\title{
Millisecond radio spikes in the decimetre band and their related active solar phenomena ${ }^{\star}$
}

\author{
B. P. Dąbrowski ${ }^{1}$, P. Rudawy ${ }^{2}$, R. Falewicz ${ }^{2}$, M. Siarkowski ${ }^{3}$, and A. J. Kus ${ }^{1}$ \\ 1 Toruń Centre for Astronomy, Nicolaus Copernicus University, 87-100 Toruń, ul. Gagarina 11, Poland \\ e-mail: [helios;ajk] @astro.uni.torun.pl \\ 2 Astronomical Institute of Wrocław University, 51-622 Wrocław, ul. Kopernika 11, Poland \\ e-mail: [rudawy; falewicz]@astro.uni.wroc.pl \\ 3 Space Research Centre, Polish Academy of Sciences, 51-622 Wrocław, ul. Kopernika 11, Poland \\ e-mail:ms@cbk.pan.wroc.pl
}

Received 5 August 2004 / Accepted 14 December 2004

\begin{abstract}
We present here a brief description of thirteen events of the narrowband solar millisecond radio spike emissions observed between February 2000 and December 2001. The total observing time was 1990.4 h, collected during the 357 observing days. The data were collected with the 15-m radio telescope and fast radiospectrograph of Torun Observatory, Poland. The dynamic spectra of the spikes were recorded in the $1352-1490 \mathrm{MHz}$ frequency band split into 46 frequency channels with temporal resolution equals to 12500 measurements per second per channel. The presented observations have probably the highest time resolution ever published. Using X-ray, UV and ground based observations we have analysed the main properties of the active phenomena correlated in time with the observed spikes. We found that probably all spikes are emitted as a result of some processes related to the solar flares. The spikes observed during the solar flares were emitted from events of various morphologies, various scenarios of evolution and various configurations of the interacting magnetic fields. All other emissions of the spikes, not correlated in time with solar flares, were observed during the slow increases of the X-ray flux leading to the flares. On the basis of the GOES database we have estimated that merely $2 \%$ of the solar flares are associated with the radio spikes.
\end{abstract}

Key words. Sun: radio radiation - Sun: flares - Sun: magnetic fields - Sun: X-rays, gamma rays

\section{Introduction}

Solar radio events with individual emissions shorter than $100 \mathrm{~ms}$ were reported for the first time by Dröge \& Riemann (1961), Elgarøy (1961) and de Groot (1962), who also introduced their common name, solar radio spikes. Despite more than forty years of investigation, millisecond radio spikes observed in the decimetre wavelength band ( $d m$ radio spikes in this paper), often emitted during the impulsive phase solar flares (Bastian et al. 1998), are one of the less understood solar phenomena (Dröge 1967, 1977; Bastian et al. 1998).

The emission process of the radio spikes and localization of their sources are still unclear. The emission of the spikes can be related to fine scale elementary processes of the magnetic field annihilation in the energy release region of the solar flares, occurring on very short time scales and on very small spatial scales (Benz 1985; Messmer \& Benz 2000) or/and to the "secondary" fragmentation of the magnetic fields directly in the radio source (Aschwanden \& Güdel 1992; Fleishman \& Melnikov 1999).

* Figures 3, 5, 9, 15, 16, 21, 23, 24 and 26 are only available in electronic form at http://www. edpsciences.org
The spatial structure and temporal changes of the sources of the radio spikes are unattainable by contemporary instruments working in visual, UV and X-ray domains. Fortunately, numerous radio instruments now have much higher temporal resolution. For this reason a complex interrelated analysis of the radio observations of the solar spikes could provide much valuable information concerning small- and short-scale physical processes as well as the main properties of the regions involved (Aschwanden \& Benz 1997; Robinson 1991; Benz et al. 2001).

In this paper we present a review of the thirteen events of the emission of numerous dm solar radio spikes, recorded by one of us (BPD) with the 15-m radio telescope and fast radiospectrograph of Torun Observatory, Poland between March 15, 2000 and October 30, 2001 (Da̧browski et al. 2002, 2003). The total observing time was $1990.4 \mathrm{~h}$, collected during the 357 observing days between February 2000 and December 2001. The observations were made in the $1352-1490 \mathrm{MHz}$ frequency band split into 46 channels, with high time resolution (12500 measurements per second and per channel) and moderate frequency resolution (3 $\mathrm{MHz}$ per channel). Using X-ray data collected by the Yohkoh Soft X-ray Telescope 
(SXT) (Tsuneta et al. 1991) and Hard X-ray Telescope (HXT) (Kosugi et al. 1991), GOES flux monitor and Interball/RF15I photometer (Sylwester et al. 2000), UV images taken with the Transition Region and Coronal Explorer (TRACE) (Wolfson et al. 1997) as well as EIT telescope on board the $\mathrm{SOHO}$ satellite (Delaboudinière et al. 1995) and ground based observations made in $\mathrm{H} \alpha$ hydrogen line in Wrocław Observatory, Poland, we have identified the most likely active solar phenomena related to some spikes and we have investigated the main morphological properties and evolution of these active phenomena.

\section{Main properties of the $\mathrm{dm}$ radio spikes}

The $\mathrm{dm}$ radio spikes are short living individual emissions of electromagnetic waves (usually of duration not longer than $100 \mathrm{~ms}$ ), recorded in very narrow frequency bands above an arbitrary chosen limit of the order of $1 \mathrm{GHz}$ (Dröge 1977). They occur often during the DCIM (decimetre, complex, highly structured radio emission) events. As a rule they appear in multi-element groups, typically up to many thousands of individual events each (Benz 1985; Bastian et al. 1998). Sometimes can one divide the groups of events into subgroups or "chains" of spikes (Güdel \& Benz 1988). The dm radio spikes can be observed with high time resolution due to their very high flux densities (of the order of 100 solar flux units, $1 \mathrm{sfu}=10^{-19} \mathrm{erg} \mathrm{s}^{-1} \mathrm{~cm}^{-2} \mathrm{~Hz}^{-1}$ ) and very high brightness temperature, greater than $10^{13}-10^{15} \mathrm{~K}$ (Bárta \& Karlický 2001; Benz 2002; Kuznetsov \& Vlasov 2003). This high brightness temperature indicates coherent mechanism of emission (Bastian et al. 1998).

The relative bandwidths of the radio spikes are of the order of a few percent and can vary significantly both from one event to another and within the same event (Fleishman \& Melnikov 1999; Benz 1986). Also Csillaghy \& Benz (1993) analysed two emissions of the spikes recorded in $1.08-1.6 \mathrm{GHz}$ and $1.0-1.35 \mathrm{GHz}$ bands and they found the average relative bandwidths of the spikes of $1.3 \%$ and $4.1 \%$, respectively. Messmer \& Benz (2000) found for radio spikes observed in the $0.87-1 \mathrm{GHz}$ band a mean relative bandwidth equal to around $0.8 \%$ and the smallest relative bandwidth of the individual spike equal to $0.17 \%$ only.

The time profiles of the spike emission was studied first by Güdel \& Benz (1990). In particular they found the Gaussian shape of the rise phase and the exponential decay phase, which was later interpreted as evidence of quasi-linear relaxation (Fleishman \& Arzner 2000). The mean characteristic times of the ascending and of the decaying parts of their profiles are comparable, even if the dispersion of the values is very broad (Mészárosová et al. 2003).

The dynamical radio-spectra of the spikes show their very fast drifts in frequency, with a wide scatter of the drift rates over positive and negative values (the negative drift is defined as a change of the observed frequency from higher to lower) but usually the negative drifts dominate. The drifts rates of the $\mathrm{dm}$ radio spikes lie between -1 and $-4 \mathrm{GHz}$ per second at $770 \mathrm{MHz}$ (Güdel \& Benz 1990), while their absolute drift rates lie between 2.2 to $4.3 \mathrm{GHz}$ per second in $2.7-3.8 \mathrm{GHz}$ band (Wang et al. 2002). However, observed drifts of the emission frequency of the individual spikes or groups of spikes can be construed not only as a result of the motion of the exciting factor through the plasma but also as a local change of the physical parameters of the emitting plasma. Millisecond radio spikes can have an arbitrary polarisation degree, ranging from $0 \%$ to $100 \%$. Usually the spikes originating near the solar limb are weakly polarized, those from the disk centre are highly polarized (Güdel \& Zlobec 1991).

The emission of radio spikes is often correlated in time with the hard X-ray (HXR) emission during the impulsive phase of the solar flares (Benz \& Kane 1986; Benz et al. 2001), however observed time delays of spike groups after the HXR peaks are of the order of a few seconds (Aschwanden \& Güdel 1992). The decimetre radio spikes have the highest association rate with flares $(95 \%)$ of all coherent radio emissions. On the contrary, only $2 \%$ of all hard X-ray events are associated with $\mathrm{dm}$ radio spikes (Aschwanden \& Güdel 1992). Unfortunately, due to an insufficient temporal resolution of the presently available $\mathrm{X}$-ray observations, it is not possible to check the temporal correlation of the individual radio spikes with short-term changes of the HXR flux.

The current data about the radio spikes are in favor of the idea of (at least) two different kinds of spike event: one class is composed of spike clusters originating in a post-flare phase and located far away from the main flare location (Benz et al. 2002) and the other is composed of the clusters appeared in the main flare phase and well correlated with other nonthermal flare emission: HXR (Aschwanden \& Güdel 1992) and microwave gyrosynchrotron emission (Fleishman et al. 2003). The sources of the first class of events, studied by Benz et al. (2002), are located far from strong magnetic field sites, while those from the second class (Fleishman et al. 2003) are related to the flares with the relatively strongest magnetic field.

Theoretical models of radio spike emission are based mainly on plasma emission and acceleration processes (Kuijpers et al. 1981; Tajima et al. 1990; Wentzel 1991; Bárta \& Karlický 2001) or on electron-cyclotron maser emission (Holman et al. 1980; Melrose \& Dulk 1982; Sharma \& Vlahos 1984; Winglee et al. 1988; Aschwanden 1990; Fleishman \& Yastrebov 1994; Fleishman \& Melnikov 1999). Spikes were interpreted also as radio emission of electrons accelerated in MHD cascading waves generated in plasma outflows from the magnetic reconnection region (Karlický et al. 1996). According to the most reliable estimations, the size of the sources of the individual radio spikes should be smaller than $200 \mathrm{~km}$, taking into account a coherent mechanism of emission (Bastian et al. 1998).

\section{Radio telescope and spectrograph}

The observations of the radio spikes used herein have been collected with the $15 \mathrm{~m}$ radio telescope of Torun Centre for Astronomy, Nicolaus Copernicus University in Toruń, Poland. The telescope has a solid parabolic dish supported on an equatorial mount and is used with the prime focus optics. The half width of the beam at $1420 \mathrm{MHz}$ is equal to $50 \mathrm{~min}$ of an arc. 
The receiver is uncooled; the flux density equivalent system temperature was around $2850 \mathrm{Jy}$.

During the observations of the dm spikes the $15 \mathrm{~m}$ telescope was connected to the fast radiospectrograph Pulsar Machine II (PSPM II) (Cadwell 1997). PSPM II is routinely used for pulsar observations with the 32-m radiotelescope of the Toruń Observatory. PSPM II has a $2 \times 64 \times 3 \mathrm{MHz}$ (polarization $\times$ channels $\times$ filter width) filter-bank built for observations of the pulsars at frequencies above $1 \mathrm{GHz}$ with very high temporal resolution and precise timing. For technical reasons the polarization of the emission of the $\mathrm{dm}$ spikes was not measured.

Dynamical radiospectrograms of the spikes were recorded in the $1352-1490 \mathrm{MHz}$ frequency band split into 46 channels each $3 \mathrm{MHz}$ wide. The time resolution of the collected data is equal to $80 \mu \mathrm{s}$, and all channels were scanned 12500 times per second. The presented observations of the radio spikes in the $\mathrm{dm}$ band probably have the highest time resolution ever obtained (Jin et al. 1986; Stähli \& Magun 1986; Allaart et al. 1990; Gary et al. 1991). Up to June 2000 we have observed the Sun simultaneously in the $1352-1490 \mathrm{MHz}$ band and at $327 \mathrm{MHz}$ with the $10 \mathrm{MHz}$ bandwidth in total power mode. We call this the $327 \mathrm{MHz}$ band. After June 2000 we made observations in the $1352-1490 \mathrm{MHz}$ band only.

\section{Evaluation of the radio observations}

The radio data were processed in order to make the sensitivities of the individual channels homogeneous but were not calibrated in absolute flux units. For subsequent automatic numerical evaluation of the main properties of the individual spikes we have freely adopted a working definition of the spike as a shortlasting increase of the flux with the peak more than 3 times above the level of the local background. Each individual radio spike was fitted with a two-dimensional elliptical Gaussian (using a non-linear least square method) and the main descriptive parameters were measured at the intensity level equal to half of the maximum: duration, bandwidth and slope (i.e. a rate of the drift in frequencies) (see Fig. 1). For reliable comparison of the radio data with other observations as well as for precise determination of the beginning, maximum and end of the radio event, we used the mean flux averaged over all channels. Evaluation of the main properties of the individual spikes was made using the data with intentionally limited temporal resolution (using an averaging filter of an arbitrarily chosen width from 0.8 up to $4 \mathrm{~ms}$, depending on the overall properties of the evaluated event).

Thirteen events of narrowband solar millisecond radio spikes were observed between March 15, 2000 and October 30, 2001. Using X-ray data collected with instruments installed on board the Yohkoh, GOES and Interball satellites, UV images taken with the TRACE and SOHO/EIT telescopes and ground based observations made in the $\mathrm{H} \alpha$ hydrogen line we identified the most likely active solar phenomena related to observed spikes. Unfortunately, the GOES, Interball and radio data used by us do not have spatial resolution. Yohkoh, TRACE and $\mathrm{SOHO}$ data have quiet good space resolution but relatively low time resolution. For this reason the only usable criterion of

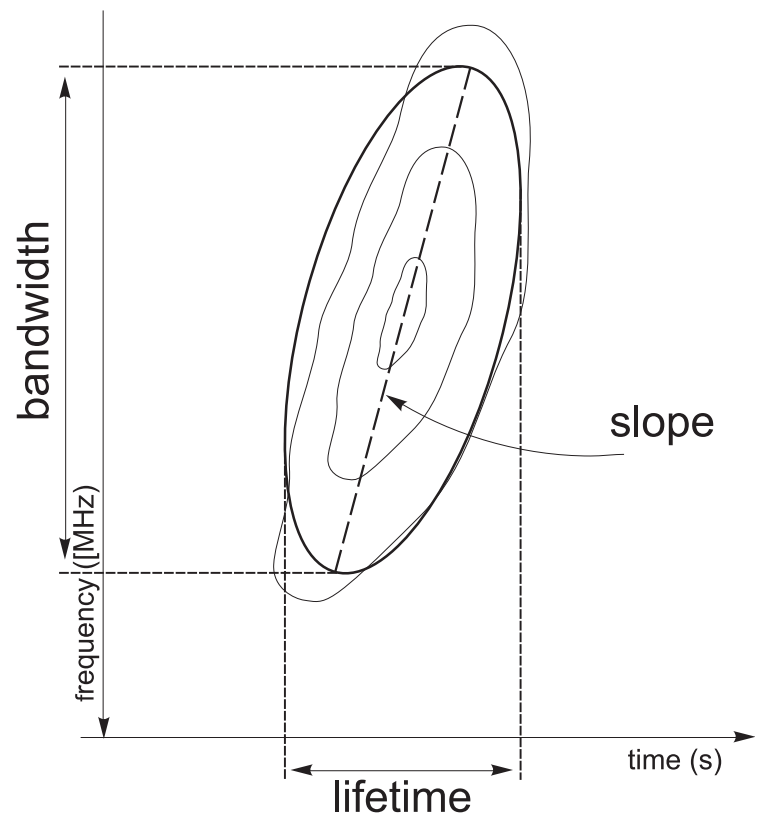

Fig. 1. Definition of the main morphological parameters of the individual $\mathrm{dm}$ radio spike evaluated using automatic non-linear fitting of the spike's dynamical radiospectrogram with a two-dimensional elliptical Gaussian.

the identification of the spikes' source was temporal correlation of some solar active phenomenon and the recording of the radio spikes. We are not able to prove undoubtedly the correctness of such a selection made in any particular case, while some cotemporal, but rather faint and undetected active processes on the Sun could also emit spikes.

We have paid special attention to precluding any artificial radio signals of a terrestrial origin. In 2000 the observations were carried out also at $327 \mathrm{MHz}$. All radio events observed in 2000 up to June 21 st and presented in this paper were observed simultaneously in both, very different, bands. The majority of the events were observed at other radio observatories, using other frequency bands. Only two presented events were recorded solely in Torun.

We have evaluated the main observational parameters of the 5328 individual $\mathrm{dm}$ radio spikes. The shortest observed spike lasted $0.0026 \mathrm{~s}$ only, the longest one $0.284 \mathrm{~s}$. The distribution of durations is right-skewed with a skewness of 1.64 , its median $0.029 \mathrm{~s}$; the mean is equal to $0.036 \mathrm{~s} .5165$ spikes (97\%) were shorter than $0.1 \mathrm{~s}$, fulfilling the commonly accepted upper limit on spike duration.

Other basic observational parameters of the spikes (drifts, widths and relative widths) shorter than $100 \mathrm{~ms}$ for each observed event are presented in Tables 1 and 2. The distributions of almost all presented parameters are right-skewed, only a few are nearly Gaussian or left-skewed. In the tables we present values of mean, median and skewness of the distribution of all parameters but we do not present the histograms.

The presented durations of the spikes are much longer than the mean duration of the spikes equal to $10 \mathrm{~ms}$, estimated for the same frequency band by Güdel \& Benz (1990), and also are 
Table 1. Main observational properties of the dm radio spikes shorter than $100 \mathrm{~ms}$, observed between February 2000 and December 2001 with the 15 -m radio telescope and fast radiospectrograph of Toruń Observatory. The drifts in frequency were calculated for spikes with inclined dynamic spectra only, vertical spectra were considered as having non-measurable drifts.

\begin{tabular}{|c|c|c|c|c|c|c|c|c|c|c|c|}
\hline & \multicolumn{6}{|c|}{2000} & \multicolumn{4}{|c|}{2001} & \multirow[b]{2}{*}{ all } \\
\hline & 15 Mar. & 26 Mar. & 17 May & 21 May & 21 Jun. & $11 \mathrm{Jul}$. & 22 Mar. & 23 Apr. & 14 May & 30 Oct. & \\
\hline No. of spik & 854 & 208 & 73 & 143 & 629 & 1403 & 174 & 576 & 876 & 392 & 5328 \\
\hline No. spikes $<100 \mathrm{~ms}$ & 817 & 156 & 62 & 143 & 597 & 1383 & 173 & 576 & 876 & 382 & 5165 \\
\hline \multicolumn{12}{|l|}{ Duration (s) } \\
\hline $\min / \max$ & $0.015 / 0.099$ & $0.025 / 0.099$ & $0.029 / 0.099$ & $0.010 / 0.046$ & $0.012 / 0.099$ & $0.009 / 0.099$ & $0.008 / 0.099$ & $0.003 / 0.071$ & $0.004 / 0.051$ & $0.005 / 0.099$ & $0.003 / 0.099$ \\
\hline $\begin{array}{l}\text { mean/median (skew.) } \\
\text { Width (MHz): }\end{array}$ & $0.058 / 0.057(0.2)$ & $0.064 / 0.064$ (0.6) & .069/0.073 (-0.5) & $0.02 / 0.018(1.4)$ & $0.046 / 0.041(0.7)$ & $0.029 / 0.024(1.9)$ & $0.037 / 0.032(0.9)$ & $.026 / 0.023(0.8)$ & $0.015 / 0.013(1.9)$ & $0.047 / 0.046(0.333)$ & $0.036 / 0.029(0.8)$ \\
\hline $\min / \max$ & $3.1 / 18.9$ & $4.2 / 20.4$ & $3.6 / 17.9$ & $3.1 / 26.4$ & $3.8 / 35.5$ & $3.1 / 31.4$ & $3.1 / 26.7$ & $3.0 / 23.8$ & $3.0 / 34.5$ & $3.1 / 25.9$ & $3.0 / 35.5$ \\
\hline mean/median (skew.) & $8.19 / 7.9(0.7)$ & $9.69 / 9.5(0.8)$ & $9.96 / 9.8(0.3)$ & $10.07 / 9.2(1.3)$ & $11.36 / 10.6(1.2)$ & $10.43 / 9.5(1.0)$ & 10.19/9.5 (0.9) & $8.47 / 8.2(0.8)$ & $9.97 / 9.0(1.2)$ & $12.30 / 12.0(0.2)$ & $10.0 / 9.2(1.1)$ \\
\hline \multicolumn{12}{|l|}{ ati } \\
\hline $\min / \max$ & $0.21 / 1.31$ & $0.29 / 1.40$ & $0.25 / 1.23$ & $0.23 / 1.92$ & $0.27 / 2.47$ & $0.21 / 2.42$ & $0.22 / 1.88$ & $0.21 / 1.65$ & $0.21 / 2.42$ & $0.22 / 1.82$ & $0.21 / 2.47$ \\
\hline mean/median (skew.) & $0.58 / 0.56(0.7)$ & $0.68 / 0.66(0.7)$ & $0.70 / 0.67(0.4)$ & $0.72 / 0.66(1.3)$ & $0.80 / 0.74(1.1)$ & $0.74 / 0.68(1.0)$ & $0.72 / 0.69(0.8)$ & $0.60 / 0.58(0.8)$ & $0.70 / 0.64(1.2)$ & $0.87 / 0.86(0.2)$ & $0.70 / 0.65(1.1)$ \\
\hline \multicolumn{12}{|l|}{ Negative } \\
\hline No. spikes & 642 & 140 & 49 & 49 & 437 & 212 & 122 & 430 & 253 & 344 & 2678 \\
\hline $\min / \max$ & $63 / 2630$ & 92/1798 & 124/1007 & $261 / 5462$ & $86 / 4976$ & $64 / 3850$ & 196/2595 & 69/12141 & $215 / 13569$ & $113 / 5491$ & $63 / 13569$ \\
\hline mean/median (skew.) & $396 / 312(2.7)$ & 307/239 (3.9) & $301 / 206(2.0)$ & $1920 / 1484(0.9)$ & 630/501 (3.1) & $1170 / 992(0.7)$ & 616/539 (2.6) & $596 / 316(6.4)$ & 2607/2257(2.1) & $462 / 347$ (6.9) & $776 / 405(4.4)$ \\
\hline \multicolumn{12}{|l|}{ Positive dri } \\
\hline no. spikes & 85 & 8 & 7 & 40 & 105 & 179 & 17 & 24 & 221 & 20 & 706 \\
\hline $\mathrm{mi}$ & $51 / 2444$ & $218 / 872$ & $87 / 1971$ & 250/9102 & $116 / 2788$ & $143 / 5155$ & $111 / 1771$ & $117 / 13425$ & 242/9525 & 236/3624 & $51 / 13425$ \\
\hline man/median (ckew) & $688 / 544(1.5)$ & $462 / 384(0.5)$ & $632 / 433(1.3)$ & $2837 / 2443(1.1)$ & $815 / 580(1.5)$ & $1219 / 1055(1.3)$ & $761 / 637(0.3)$ & $1752 / 719(3.0)$ & $2607 / 2415(1.2)$ & $1157 / 1195(1.1)$ & $1612 / 1105(2.3)$ \\
\hline
\end{tabular}


Table 2. Main properties of the selected dm radio spikes shorter than $100 \mathrm{~ms}$ observed in three events on 11 July 2000 . The drifts in frequency were calculated for spikes with inclined dynamic spectra only, vertical spectra were considered as having non-measurable drifts.

\begin{tabular}{l|rrr}
\hline \hline & \multicolumn{3}{|c}{11 July 2000 } \\
\cline { 2 - 4 } & $12: 30: 10-13: 30: 10$ & $14: 34: 50-14: 51: 30$ & $14: 57: 40-15: 02: 20$ \\
\hline No. of spikes & 826 & 237 & 340 \\
No. spikes $<100 \mathrm{~ms}$ & 808 & 237 & 338 \\
Duration (s) & & & \\
min/max & $0.011 / 0.99$ & $0.012 / 0.097$ & $0.009 / 0.085$ \\
mean/median (skew.) & $0.033 / 0.027(1.7)$ & $0.025 / 0.019(2.1)$ & $0.022 / 0.017(2.5)$ \\
Width (MHz): & & & \\
min/max & $3.1 / 31.4$ & $3.9 / 25.6$ & $3.1 / 30.7$ \\
mean/median (skew.) & $10.1 / 9.1(1.1)$ & $13.57 / 13.4(0.3)$ & $9.09 / 8.2(1.4)$ \\
Relative width (\%) & & & \\
min/max & $0.22 / 2.16$ & $0.28 / 1.77$ & $0.21 / 2.24$ \\
mean/median (skew.) & $0.71 / 0.65(1.1)$ & $0.96 / 0.93(0.3)$ & $0.64 / 0.57(1.4)$ \\
Negative drifts (MHz/s) & & & 40 \\
No. spikes & 140 & 32 & $254 / 2622$ \\
min/max & $64 / 3850$ & $330 / 2952$ & $1160 / 1058(0.4)$ \\
mean/median (skew.) & $1105 / 799(0.9)$ & $1467 / 1512(0.0)$ & 38 \\
Positive drifts (MHz/s) & & & $144 / 2423$ \\
No. spikes & 105 & 36 & $894 / 672(0.9)$ \\
min/max & $142 / 3853$ & $373 / 5155$ & \\
mean/median (skew.) & $1174 / 966(1.1)$ & $1695 / 1680(1.2)$ & \\
\hline
\end{tabular}

Table 3. Main properties of the dm radio spikes with and without internal structure, shorter than $100 \mathrm{~ms}$, observed between February 2000 and December 2001 with the 15-m radio telescope and fast radiospectrograph of Torun Observatory.

\begin{tabular}{l|rr}
\hline \hline & Spikes without internal structure & Spikes with internal structure \\
\hline No. spikes & 1699 & 1837 \\
Duration (s) & & \\
min/max & $0.008 / 0.099$ & $0.003 / 0.099$ \\
mean/median (skew.) & $0.029 / 0.024(1.9)$ & $0.046 / 0.047(0.1)$ \\
Width (MHz): & & \\
min/max & $3.1 / 31.4$ & $3.0 / 25.9$ \\
mean/median (skew.) & $10.38 / 9.5(1.0)$ & $9.2 / 8.7(0.9)$ \\
Relative width (\%) & & \\
min/max & $0.21 / 2.24$ & $0.21 / 1.82$ \\
mean/median (skew.) & $0.73 / 0.68(1.0)$ & $0.65 / 0.61(0.9)$ \\
Negative drifts (MHz/s) & & 1462 \\
No. spikes & 381 & $63 / 12141$ \\
min/max & $64 / 5462$ & $467 / 321(9.1)$ \\
mean/median (skew.) & $1089 / 762(1.7)$ & 136 \\
Positive drifts (MHz/s) & & $51 / 13425$ \\
No. spikes & 236 & $942 / 604(6.1)$ \\
min/max & $111 / 9102$ & \\
mean/median (skew.) & & \\
\hline
\end{tabular}

longer than the $10 \mathrm{~ms}$ mean duration of the spikes observed by Mészárosová et al. (2003). The reason for the difference is not clear, but it cannot be attributed to the observational setup used, for example to the limited temporal resolution of the collected data.

A part of the observed spikes, called by us "spikes without internal structure", have a simple form of a single increase of the radio flux, limited in time and frequency (see Fig. 22). We have also recorded numerous short, spike-like $\mathrm{dm}$ radio emissions showing a well outlined subtle internal structure, consisting of a few local, internal maxima (see Fig. 19). We will refer them arbitrary as "spikes with internal structure".
For single-frequency instruments or instruments with a limited temporal resolution both kinds of spikes look identical. If the spikes with internal structure are genuine individual spikes with real internal subtle structure or if they are in fact very shortlasting chains of tightly packed spikes with very fast drifts in frequencies should be addressed by future investigations. The main properties of the $\mathrm{dm}$ radio spikes with and without internal structure and shorter than $100 \mathrm{~ms}$, observed by us between February 2000 and December 2001, are presented in Table 3.

We classified five radio events (observed on 21 May 2000, 11 July 2000 and 22 March 2001) as ones with spikes without the internal structure only. These events contained 1720 spikes. 
The distribution of the durations of the 1699 spikes shorter than $0.1 \mathrm{~s}$ is right-skewed with skewness equal to 1.86 , its median is equal to $0.024 \mathrm{~s}$ and its mean is equal to $0.029 \mathrm{~s}$. Four radio events (observed on 15 March 2000, 17 May 2000, 23 April 2001 and 30 October 2001) contained 1895 spikes with internal structure, with 1837 spikes shorter than $0.1 \mathrm{~s}$. The distribution of their durations is close to Gaussian (skewness equal to 0.12) with the median equal to $0.047 \mathrm{~s}$ and the mean equal to $0.046 \mathrm{~s}$. The time scale of the internal subtle structures is roughly one order of magnitude shorter. The relative widths in frequency of these emissions are similar to the parameters of the "classical" spikes without internal structure.

The drifts of the spikes with internal structure are distinctly slower than ones without structure. The distribution of the negative drifts of the 381 spikes without structure shorter than $0.1 \mathrm{~s}$ is right-skewed with skewness equal to 1.7 , its mean is equal to $1089 \mathrm{MHz} / \mathrm{s}$ and its median is equal to $762 \mathrm{MHz} / \mathrm{s}$. The distribution of the positive drifts of the 236 spikes without structure shorter than $0.1 \mathrm{~s}$ is right-skewed with skewness equal to 2.3 , its mean is equal to $1460 \mathrm{MHz} / \mathrm{s}$ and its median is equal to $1113 \mathrm{MHz} / \mathrm{s}$. The distribution of the negative drifts of the 1462 spikes with internal structure shorter than $0.1 \mathrm{~s}$ is right-skewed with skewness equal to 9.1 , its mean is equal to $467 \mathrm{MHz} / \mathrm{s}$ and its median is equal to $321 \mathrm{MHz} / \mathrm{s}$. The distribution of the positive drifts of the 136 spikes with structure and shorter than $0.1 \mathrm{~s}$ is right-skewed with skewness equal to 6.1 , its mean is equal to $942 \mathrm{MHz} / \mathrm{s}$ and its median is equal to $604 \mathrm{MHz} / \mathrm{s}$.

We have found that in a framework of the single radio event all emitted $\mathrm{dm}$ radio spikes are rather similar. Nevertheless, both types of spikes (with or without internal structure) can be emitted in the same event. Each spike has its own positive (i.e. from low to high) or negative (i.e. from high to low) drift in frequency of various speeds (from tens of $\mathrm{MHz}$ per second up to greater than the highest measurable with our instrument, equal to $37.5 \mathrm{GHz}$ per second). Assuming a plasma emission mechanism as a source of the observed spikes, the positive drift in frequency can be interpreted as a motion of the exciter downward (or local increase of the plasma density), while the negative drift can be caused by upward motion (or local decrease of the plasma density).

On the dynamic radiospectrograms of the investigated events we have noticed two kinds of well-outlined complex structures formed by numerous individual spikes: well-known chains of spikes and distinctly different structures, "columns". The chains of spikes, observed already e.g. by Güdel \& Benz (1988), are formed by tight groups of spikes with their emission band drifting in frequency (i.e. chains with drift) (see Fig. 17) or emitted in a constant band (chains without drift) (see Fig. 11). The columns of the spikes are formed by numerous individual spikes, recorded over a very short time interval on various frequency of the whole observed band (see Fig. 7). In the column, each individual spike can show positive (i.e. from low to high) or negative (i.e. from high to low) drift in frequency. The durations of the chains of the spikes observed by us vary from $100 \mathrm{~ms}$ up to $500 \mathrm{~ms}$ while the durations of the columns were not longer than $200 \mathrm{~ms}$.

\section{Description of the observed events}

We have detected thirteen radio events with eighteen emissions of groups of $\mathrm{dm}$ radio spikes. Using the Yohkoh/HXT flux curves made in L and M1 channels, we have found that eleven groups of spikes were emitted during increases of the $\mathrm{X}$-ray flux or during the impulsive phases of the solar flares, two groups were emitted during the peaks of the flares and five groups were observed during the gradual phases of the flares (according to the GOES flux curves for 1-8 $\AA$ band). The detailed descriptions of the relevant solar events are given below.

\subsection{Events with emission of dm radio spikes during the impulsive phase of the flare}

\subsubsection{March 2000}

The $\mathrm{dm}$ radio spikes were recorded between 12:20:06 UT and 12:21:08 UT (see Fig. 3) during the DCIM radio event (complex, highly structured emission between 300 and $3000 \mathrm{MHz}$ ), observed by us since 12:19:50 UT (it started before the beginning of our observations). The event peaked at 12:20:50 UT and ended at 12:26:20 UT. It was observed by us simultaneously in the $1352-1490 \mathrm{MHz}$ and $327 \mathrm{MHz}$ bands. We have recorded both types of spikes: with and without internal structure. The spikes occurred during the impulsive phase of the C5 class solar flare in the NOAA 8906 active region (S18W19) of the magnetic type $\beta-\delta$. The flare started at 12:13 UT, reached a maximum at about 12:29 UT and ended at 12:55 UT, according to the GOES classification. A weakly marked plateau on the GOES flux curve preceded the impulsive phase of the flare (see Fig. 2). The HXT telescope on board the Yohkoh telescope recorded the emission of the flare in channel L (14-23 keV), which peaked at 12:20 UT, six seconds before the beginning of the emission of the spikes. Such a delay between the peak of the high energy hard X-ray emission and the beginning of the emission of the radio spikes is a typical phenomenon (Aschwanden \& Güdel 1992). The maximum of the total radio flux was observed about $50 \mathrm{~s}$ after the maximum of the HXR emission.

The flare was caused very likely by the Y- or I-type interaction of the loops in an arcade of loops (Sakai \& de Jager 1996), well visible on the images taken with the SXT telescope (see Fig. 4). The maximum of the temperature of the plasma $T=8 \mathrm{MK}$ was recorded at 12:25 UT, while the maximum of the emission measure $E M=4 \times 10^{48} \mathrm{~cm}^{-3}$ occurred four minutes later (both values were evaluated using GOES data).

\subsubsection{June 2000}

A solar radio burst was observed between 09:24:00 UT and 09:30:08 UT simultaneously in the 1352-1490 $\mathrm{MHz}$ and $327 \mathrm{MHz}$ bands. During the long-lasting peak of the event numerous dm radio spikes were recorded between 09:24:59 UT and 09:26:05 UT (see Fig. 5). We noticed both types of spikes: with and without internal structure, some of them aggregated in the columns of the spikes. The radio spikes were observed at the end of the impulsive phase of the M1.3 class solar 

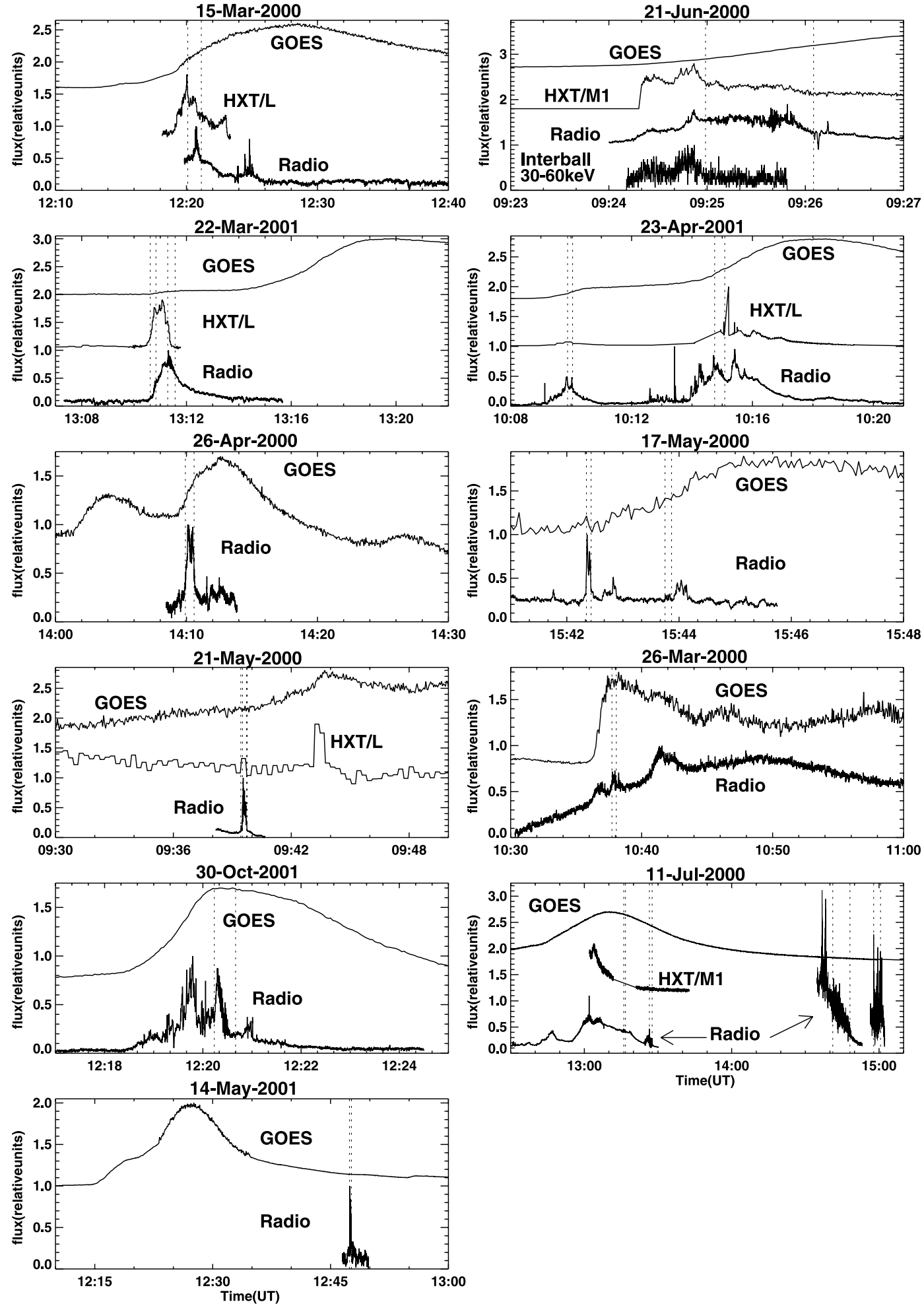

Fig. 2. GOES (1-8 ̊), HXT/L (14-23 keV), HTX/M1 (23-33 keV), Interball (30-60 keV) and radio (1352-1490 MHz) fluxes recorded during the investigated radio events with $\mathrm{dm}$ radio spikes. The time range of the dm radio spike emission is outlined by vertical dotted lines. The $y$-axis is scaled in relative units.

flare, which started at 09:22 UT, reached a maximum at about 09:29 UT, and ended at 09:37 UT (see Fig. 2). The flare occurred in the NOAA 9042 active region (N24W34) of magnetic type $\beta-\gamma$ (Rudawy et al. 2002). It was observed also as a SF $\mathrm{H} \alpha$ class flare between 09:24 UT and 09:41 UT.
In the maximum of the flare the plasma temperature was equal to $T=15.5 \mathrm{MK}$ at $09: 27 \mathrm{UT}$ and the emission measure was $E M=1.5 \times 10^{49} \mathrm{~cm}^{-3}$ at 09:33 UT (both values are evaluated using GOES data). Immediately after the impulsive phase a long-lasting flow of the plasma started along a system of 


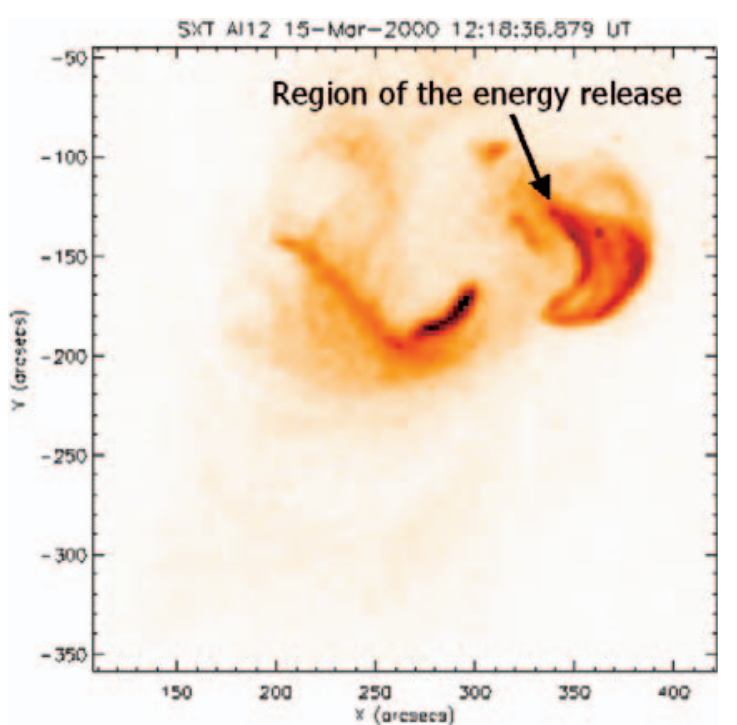

Fig. 4. Image of the NOAA 8906 active region taken with SXT/A112 on 15 March 2000 at 12:18:37 UT, one and a half minutes before the emission of the $\mathrm{dm}$ radio spikes.

stretched magnetic loops (see Fig. 6). Temporal evolution of the system of loops also indicates a co-temporal eruption of an active region filament.

\subsubsection{March 2001}

The radio DCIM event was observed between 13:10:28 UT and 13:13:45 UT. The first group of dm radio spikes was recorded between 13:10:37 UT and 13:10:50 UT and the second one between 13:11:17 UT and 13:11:34 UT (see Fig. 7). On the dynamic radiospectrogram the spikes forms columns, the individual spikes have no internal structure. Both groups of spikes occurred at the beginning and at the end of the impulsive phase of the M1.0 solar flare, respectively (see Fig. 2). The M1.0 solar flare started at 13:12 UT, peaked at 13:19 UT and end at 13:34 UT. It was observed in the NOAA 9390 active region (N14E60) of magnetic type $\beta$. The start of the impulsive phase of the flare was marked by the temporal increase of the hard X-ray flux recorded with the HXT/L telescope. The GOES X-ray light curve of the flare shows a plateau following the impulsive phase. The maximum of the temperature of the plasma $T=16.1 \mathrm{MK}$ was observed at 13:17 UT, while the maximum of the emission measure $E M=1.1 \times 10^{49} \mathrm{~cm}^{-3}$ was recorded at 13:28 UT (both values are evaluated using GOES data). During the maximum of the flare, the TRACE telescope recorded bright loops and bright emission kernels (in the $171 \AA$ band) but we have not found any bulk mass motion or severe rebuilding of the magnetic configuration (see Fig. 8).

\subsubsection{April 2001}

The radio DCIM event started at 10:08:30 UT and ended at 10:21:50 UT. The first group of $\mathrm{dm}$ radio spikes was recorded between 10:09:53 UT and 10:10:03 UT, the second one between 10:14:45 UT and 10:15:05 UT (see Fig. 9). We have recorded both types of spikes: with and without internal

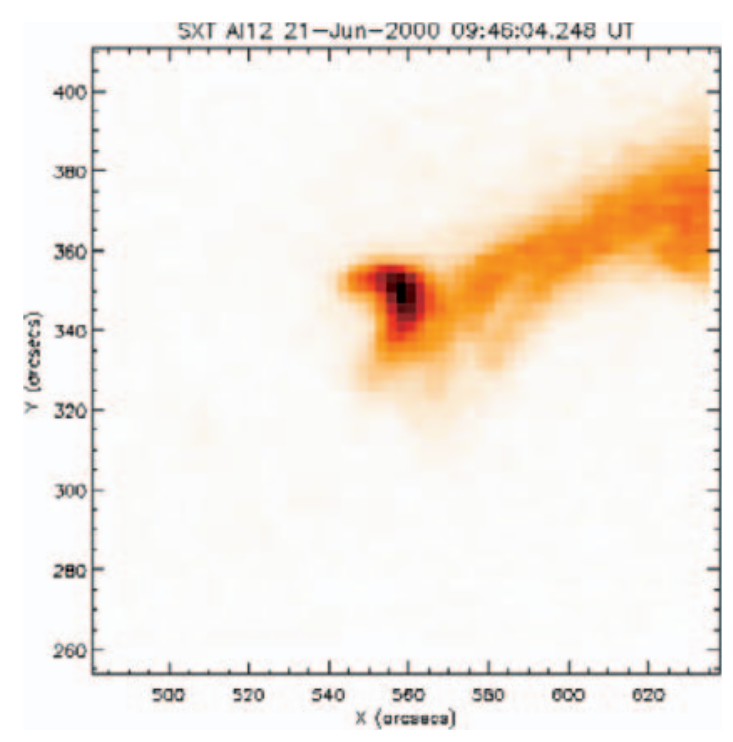

Fig. 6. Image of the NOAA 9042 active region taken with SXT/A112 on 21 June 2000 at 09:46:04 UT.

structure. The first group of spikes, well correlated in time with a short emission of hard X-rays, occurred at the beginning of the minor increase of the X-ray flux, followed by the plateau on the X-ray light curve of the C9.1 solar flare (see Fig. 2). The flare occurred in the NOAA 9433 active region (N17E26) of magnetic type $\beta-\gamma-\delta$; it started at 10:06 UT, peaked at 10:18 UT and ended at 10:23 UT. The second group of spikes was recorded at the beginning of the main impulsive phase of the flare which was signalled also by the second increase of the hard X-ray flux. The same flare was observed as an SF $\mathrm{H} \alpha$ class flare between 10:10 UT and 10:29 UT. The maximum of the temperature of the plasma $T=14.6 \mathrm{MK}$ was recorded at 10:17 UT, while the maximum of the emission measure $E M=1.1 \times 10^{49} \mathrm{~cm}^{-3}$ at 10:21 UT (both values are evaluated using GOES data). Both groups of spikes occurred simultaneously with the flow of matter along arcades of the loops (see Fig. 10).

\subsection{Events with emission of $d m$ radio spikes during the increase phase of the flare, outside the impulsive phase.}

\subsubsection{April 2000}

The dm radio spikes were recorded between 14:09:55 UT and 14:10:35 UT (see Fig. 11), during the radio event observed from 14:09:34 UT, which peaked at 14:10:08 UT and ended at 14:11:08 UT. The event was observed by us simultaneously in the $1352-1490 \mathrm{MHz}$ band and in the $327 \mathrm{MHz}$ band, where we recorded a noise storm. While the fluxes of the individual spikes were very low and the spikes were very short in time, we are not able to make reliable quantitative measurements of their main properties, except the mean duration of $0.05 \mathrm{~s}$, and a bandwidth about $7 \mathrm{MHz}$. Some spikes formed the separate chains of the spikes. The spikes were observed during a small increase of the X-ray flux (B7 GOES class), observed in X-rays between 14:09 UT and 14:26 UT, mainly with the 


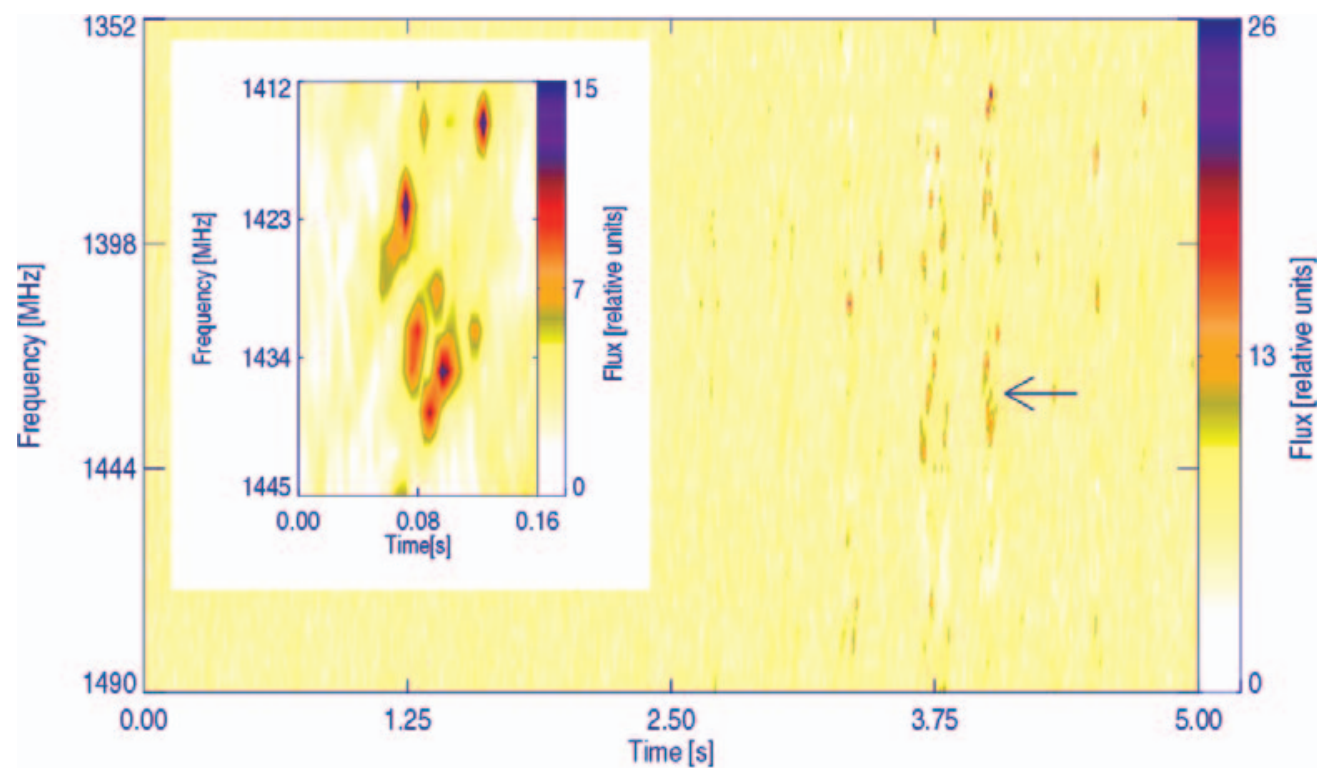

Fig. 7. Dynamic radiospectrogram of the dm radio spikes recorded on 22 March 2001, beginning at 13:11:15 UT. On the spectra one can recognise columns of spikes. The individual spikes have no internal structure.
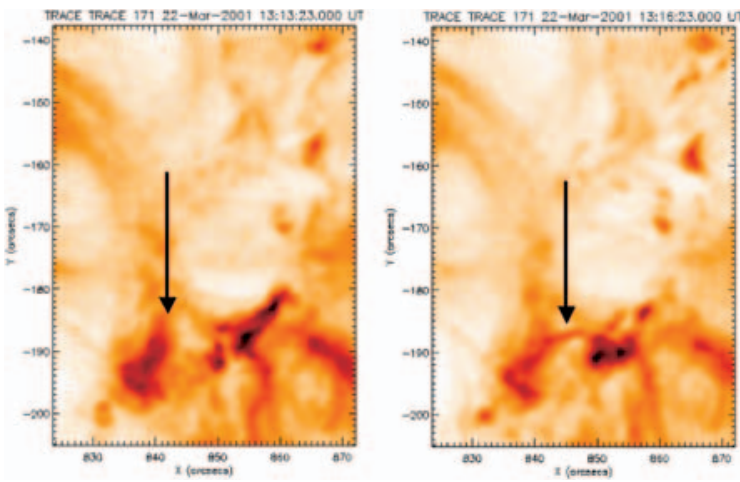

Fig. 8. Image of the NOAA 9390 active region taken with TRACE (171 ̊) telescope on 22 March 2001 at 13:13:23 UT (left panel) and at 13:16:23 UT (right panel). The arrows points to the loop gradually filled by the hot plasma.

hard channel $(0.5-4 \AA)$, but also in the soft channel (1-8 $)$ of the GOES satellite (see Fig. 2). This increase of the X-ray flux was caused by an I-type interaction of two loops, well recorded by the SXT telescope in the NOAA 8970 active region (S15E10) of the magnetic type $\beta-\gamma$ (see Fig. 12). The beginning of the emission of the radio spikes apparently occurred during the energy release in the system of interacting loops. The maximum of the temperature of the plasma $T=9 \mathrm{MK}$ occurred at 14:11 UT, while the maximum of the emission measure $E M=4 \times 10^{47} \mathrm{~cm}^{-3}$ was observed two minutes later (the values are evaluated using SXT data).

\subsubsection{May 2000}

The $\mathrm{dm}$ radio spikes occurred in two groups, separated in time by nearly $79 \mathrm{~s}$ (see Fig. 13). The first group of spikes was recorded between 15:42:21 UT and 15:42:26 UT, the second one was recorded between 15:43:45 UT and 15:43:52 UT.

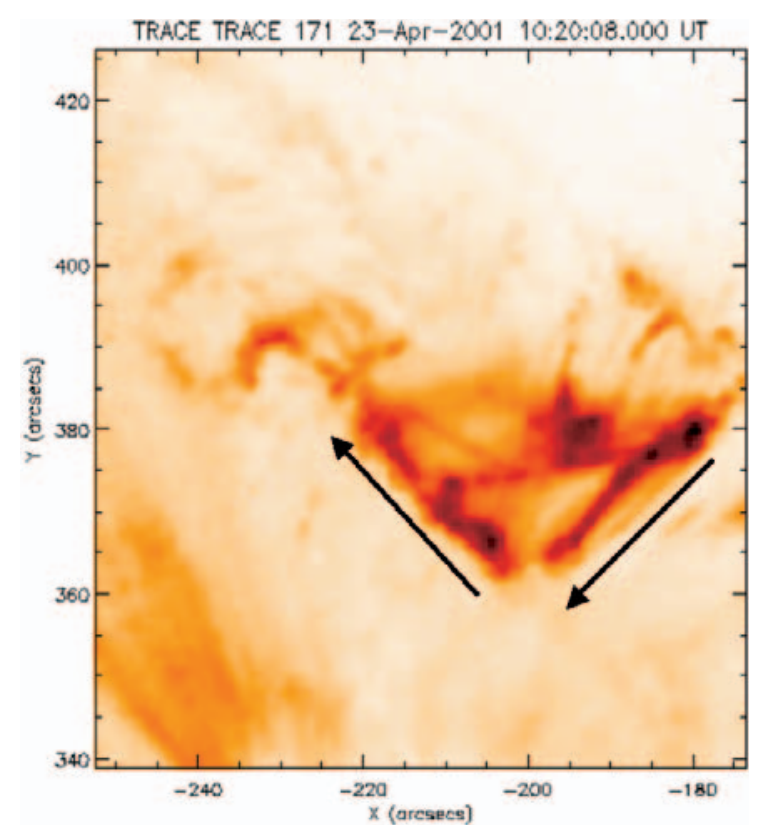

Fig. 10. Image of the NOAA 9433 active region taken with TRACE (171 A) on 23 April 2001 at 10:20:08 UT. The arrows show the directions of the plasma motion.

The whole radio event, which started at 15:41:40 UT, consists of a few local maxima with the highest one exactly co-temporal with the first group of $\mathrm{dm}$ radio spikes mentioned before. It was observed by us simultaneously in the $1352-1490 \mathrm{MHz}$ band and in the $327 \mathrm{MHz}$ band, where we recorded the noise storm. The spikes were well distinguishable from the recorded radio background. We have recorded both kinds of the spikes: with and without internal structure. The radio spikes were observed during the small GOES X-ray event (C2.2 GOES class) (the first group of spikes occurred at the beginning of the X-ray flux rise) in the NOAA 8999 active region (N20E20) of magnetic 


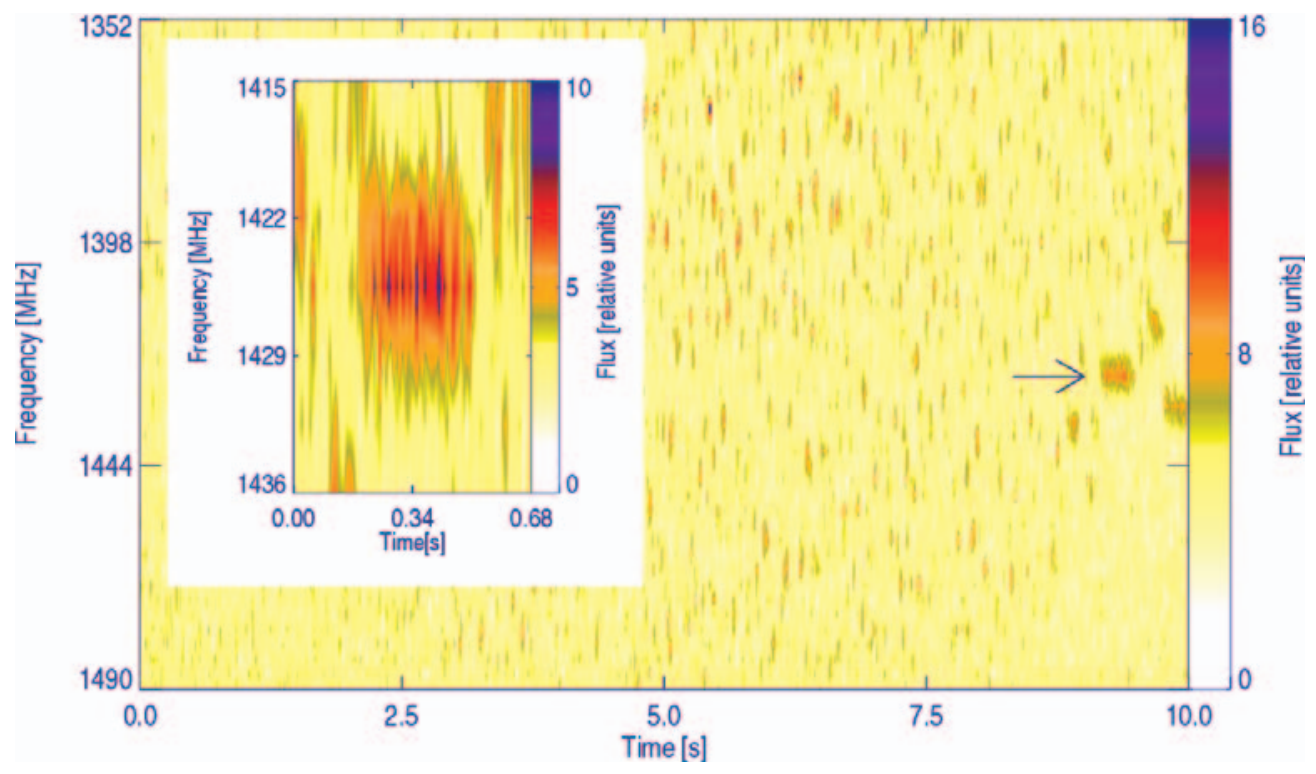

Fig. 11. Dynamic radiospectrogram of the dm radio spikes recorded on 26 April 2000, beginning at 14:10:05 UT. Some spikes formed separate chains.

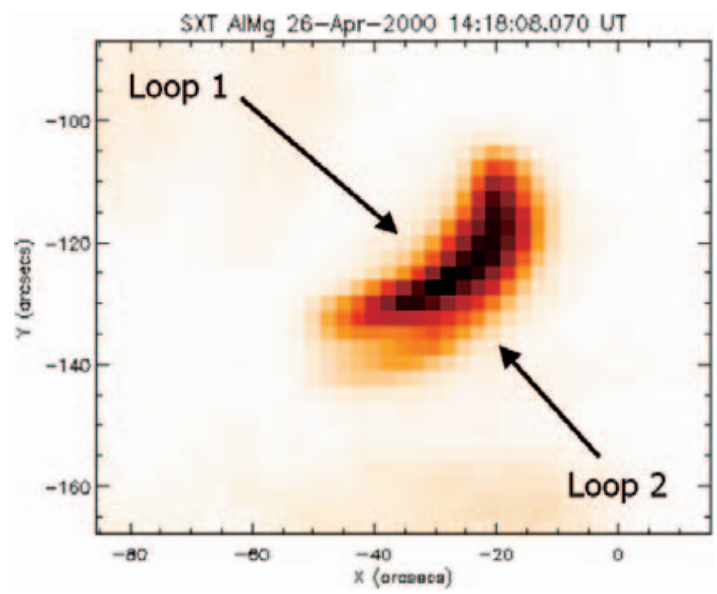

Fig. 12. Image of the NOAA 8970 active region taken with SXT/AlMg telescope on 26 April 2000 at 14:18:08 UT.

type $\beta$ (see Fig. 2). The X-ray event was associated with an SF class $\mathrm{H} \alpha$ flare reported between 15:44 UT and 15:56 UT. The images of the active region taken with the TRACE telescope in the $195 \AA$ band shows two separate regions of interaction of the short loops and an ejection of the plasma from the northern part of the active region, which was co-temporal with the emission of the dm radio spikes (see Fig. 14).

\subsubsection{May 2000}

The whole radio event started at 09:39:14 UT, reached maximum at 09:39:33 UT and ended at 09:40:10 UT. It was observed by us simultaneously in the $1352-1490 \mathrm{MHz}$ and $327 \mathrm{MHz}$ bands. During this event we recorded two groups of $\mathrm{dm}$ radio spikes: the first one started at 09:39:26 UT and ended at 09:39:31 UT, the second one started at 09:39:44 UT and ended 09:39:45 UT (see Fig. 15). The spikes have no the internal structure. Between the observations of both groups of spikes we also recorded a radio emission with the dynamic radiospectrograms similar to the "glued" spikes. The radio spikes were observed during the more than $1.5 \mathrm{~h}$ very slow increase of the X-ray signal (the GOES signal was at the C6 level during the emission of the dm radio spikes), which peaked at about 10:25 UT at the C8.2 level as an SF class solar flare in the NOAA 8996 active region (S18W47) of magnetic type $\beta$ (see Fig. 2). We have not found any other manifestations of solar activity which can be combined with the spikes.

\subsection{Events with emission of $d m$ radio spikes during the peak of the flare}

\subsubsection{March 2000}

The radio spikes were observed from 10:37:44 UT to 10:38:04 UT, during the DCIM radio event observed by us from 10:30:20 UT to 10:50:00 UT (see Figs. 16 and 17) simultaneously in 1352-1490 MHz and $327 \mathrm{MHz}$ bands. The recorded radio flux consists of two components: quiet rise and fall emission overlaid with three short-lasting peaks. The emission of the spikes occurred at the same time as the second, but not the highest, peak of the radio flux (see Fig. 2). Numerous recorded spikes have internal structure but other have not. Some spikes were aggregated in chains. The spikes occurred during the maximum of the C2 class solar flare, which started at 10:35 UT, reached the maximum at 10:38 UT, and ended at 10:44 UT (according to the GOES classification). The flare was observed in the NOAA 8941 active region (S18E09) of magnetic type $\beta$. The maximum of the temperature of the plasma $T=7 \mathrm{MK}$ occurred at 10:36 UT, while the maximum of the emission measure $E M=2 \times 10^{47} \mathrm{~cm}^{-3}$ was at 10:44 UT (both values are evaluated using GOES data). Between 10:36:51 UT and 10:38:04 UT TRACE (195 ^) recorded a spray ejected from the central part of the active region. The average velocity of the 


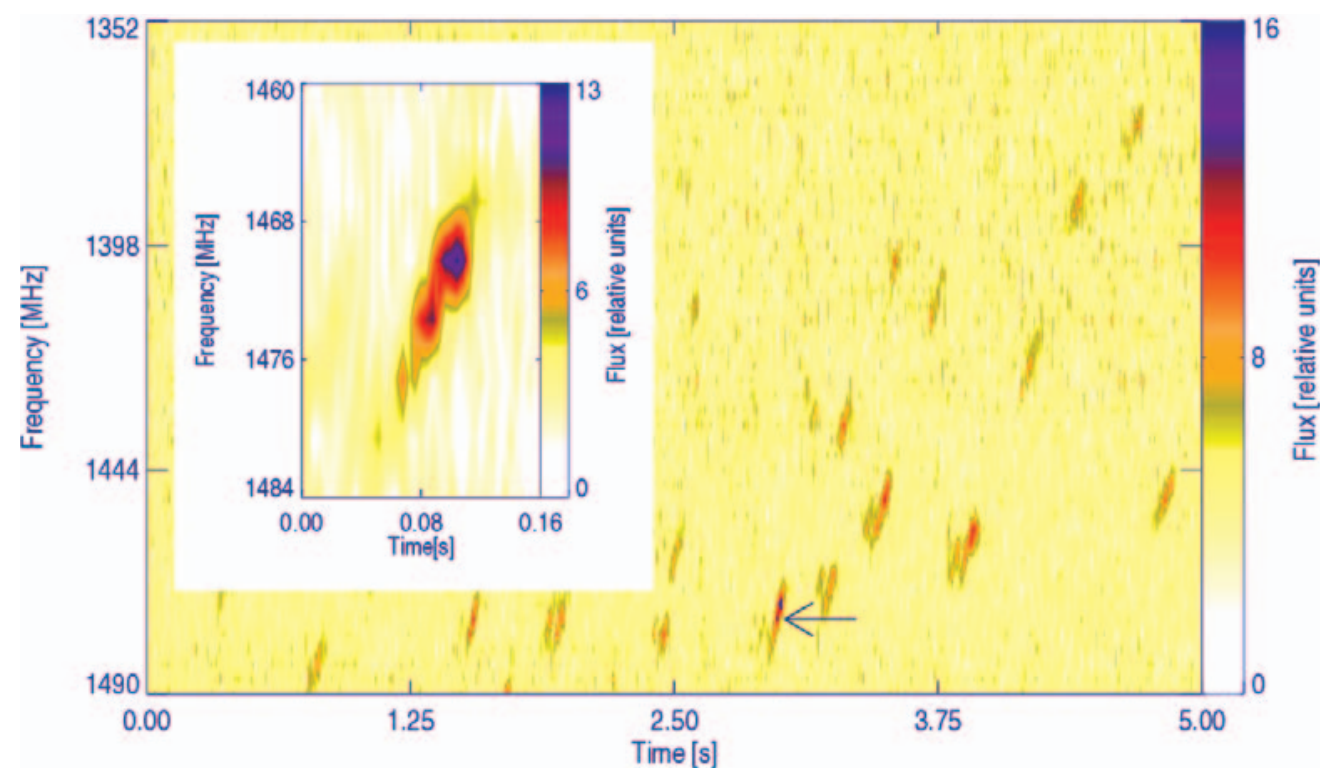

Fig. 13. Dynamic radiospectrogram of the dm radio spikes recorded on 17 May 2000, beginning at 15:43:45 UT.

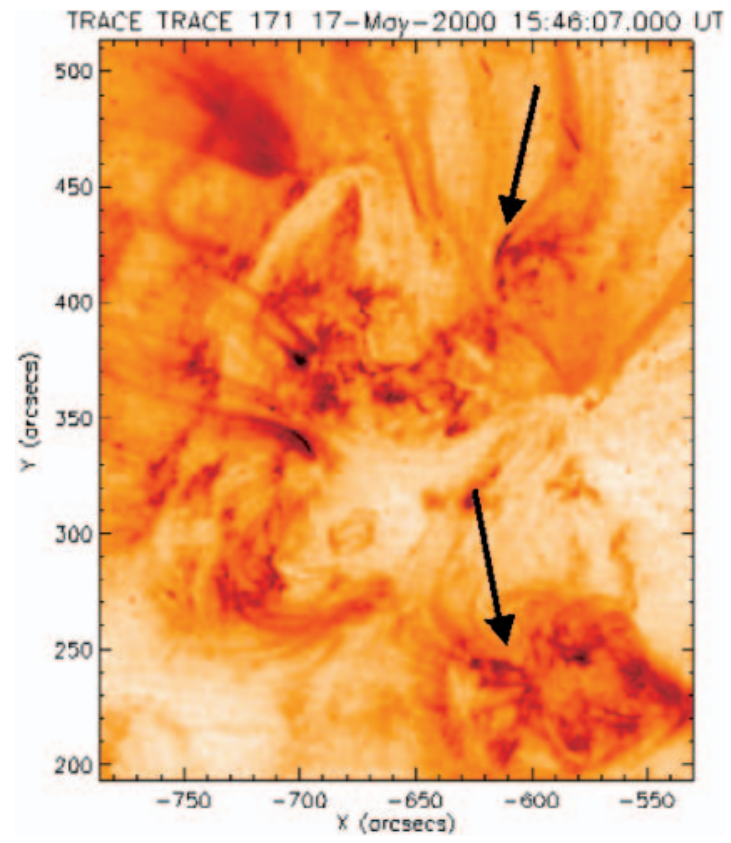

Fig. 14. Image of the NOAA 8999 active region taken with the TRACE $(171 \AA)$ telescope on 17 May 2000 at 15:46:07 UT. The arrows points to the regions with clearly visible motions of matter along the magnetic loops.

spray was $650 \mathrm{~km} \mathrm{~s}^{-1}$ (see Fig. 18). Radio spikes were released at the same time as the spray was observed.

\subsubsection{October 2001}

The radio DCIM event started at 12:18:14 UT and ended at 12:22:36 UT. The dm radio spikes were recorded between 12:20:14 UT and 12:20:40 UT, during the peak of the C7.4 solar flare (start at 12:16 UT, maximum at 12:20 UT, end at 12:23 UT) in the NOAA 9682 active region (N12E10) of magnetic type $\beta$, noted also as a $1 \mathrm{~N}$ class $\mathrm{H} \alpha$ flare between
12:18 UT and 12:32 UT (see Figs. 19, 2). The maximum of the temperature of the plasma $T=12.3 \mathrm{MK}$ occurred at 12:20 UT, while the maximum of the emission measure $E M=8 \times 10^{48} \mathrm{~cm}^{-3}$ was reached at 12:21 UT (both values are evaluated using GOES data). The observed radio spikes have internal structure and form chains. They occurred during the bulk motion of the plasma along the long loops, observed with the TRACE telescope in the $171 \AA$ band (see Fig. 20).

\subsection{Events with emission of $d m$ radio spikes during the gradual phase of the flare}

\subsubsection{July 2000 (three separate events)}

On 11 July 2000 we recorded four groups of radio spikes emitted during three separate radio events (see Figs. 21-24, respectively). The first event consisted of two separate groups of spikes, recorded by us during the DCIM-type radio event observed between 12:30:10 UT and 13:30:10 UT. The first group was recorded between about 13:16:03 UT and 13:16:44 UT, the second one between about 13:26:30 UT and 13:27:37 UT. The second group was observed also by Karlický and co-workers, who reported dm radio spikes between 13:25:40 UT and 13:27:40 UT in the frequency band 1.0-1.8 GHz (Karlický et al. 2001). The third group of spikes was recorded from 14:41:10 UT to 14:48:10 UT, during the next DCIM radio event observed by us between 14:34:50 UT and 14:51:30 UT. It started before the start of our observations. The last group of spikes, observed from 14:57:49 UT to $15: 00: 37 \mathrm{UT}$, occurred during the last radio event, observed from 14:57:40 UT to 15:02:20 UT.

All recorded spikes have no internal structure. The main properties of the spikes are presented in Table 2. The emission of the all groups of the spikes occurred during the X1.0 class solar flare, which started at 12:12 UT and reached the maximum at 13:10 UT in the NOAA 9077 active region (N18E27) of magnetic type $\beta-\gamma-\delta$ (see Fig. 2). The first two groups 


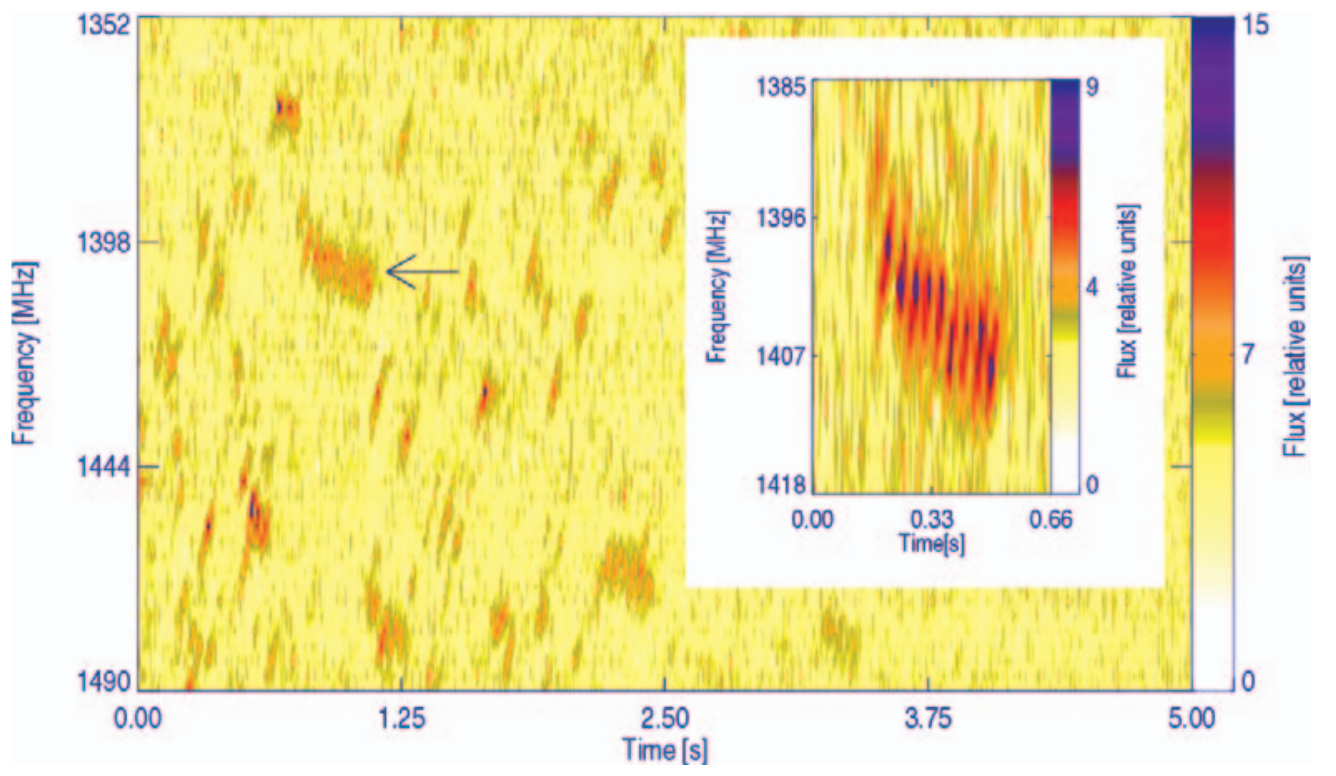

Fig. 17. Dynamic radiospectrogram of the dm radio spikes recorded on 26 March 2000, beginning at 10:38:00 UT. Some spikes form clearly visible chains with drift in frequency.

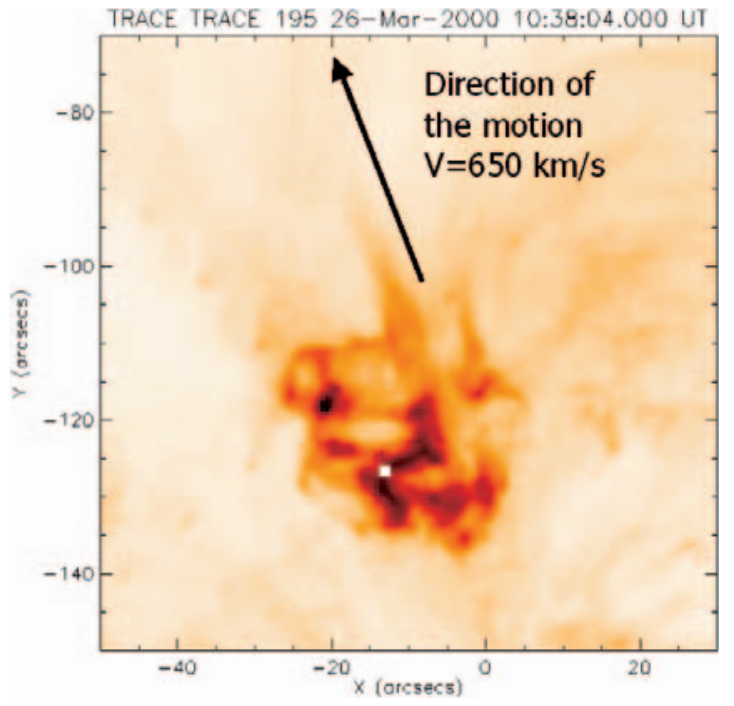

Fig. 18. Image of the NOAA 8921 active region taken with TRACE $(195 \AA)$ on 26 March 2000 at 10:38:04 UT.

of spikes occurred after the maximum of this flare, the other during its gradual phase. The images of the active region taken with the SXT/Be119 telescope after 13:06 UT show a Y-type interaction of the magnetic loops (see Fig. 25). These loops probably form the main region of energy release of the flare. The maximum of the temperature of the plasma $T=19.2 \mathrm{MK}$ occurred at 12:16 UT, while the maximum of the emission measure $E M=1.1 \times 10^{50} \mathrm{~cm}^{-3}$ at 13:17 UT (both values are evaluated using GOES data). Very likely the first, third and fourth groups of spikes were emitted from this active region. An identification of the source of the second group of spikes is problematic, while this group of spikes (recorded between about 13:26:30 UT and 13:27:37 UT) is well correlated in time with an SF class $\mathrm{H} \alpha$ flare, observed between 13:25 UT and 13:29 UT in the NOAA 9069 active region (S17W39) of magnetic type $\beta$.

\subsubsection{May 2001}

The radio event started at 12:47:25 UT, reached a maximum at 12:47:27 UT and ended at 12:47:45 UT. The radio spikes were recorded between 12:47:25 UT and 12:47:39 UT (see Fig. 26), during the gradual phase of the $\mathrm{C} 2.3$ class solar flare (start at 12:13 UT, maximum at 12:27 UT) in the NOAA 9455 active region (S15W15) of magnetic type $\beta-\gamma$ (see Fig. 2). We have recorded both types of spikes: with and without internal structure. The maximum of the temperature of the plasma $T=10 \mathrm{MK}$ occurred at 12:22 UT, while the maximum of the emission measure $E M=3.6 \times 10^{48} \mathrm{~cm}^{-3}$ at 12:29 UT (both values are evaluated using GOES data). UV images of the active region taken with the TRACE telescope in the $171 \AA$ band show numerous interactions between two systems of magnetic loops of different length as well as some ejections of the plasma along the long loops heading outside the region of interactions. The ejections were co-temporal with the emission of the dm radio spikes (see Fig. 27).

\section{Discussion and conclusions}

In this paper we have described thirteen event, in which we recognized eighteen groups of $\mathrm{dm}$ radio spike emission, composed of various numbers of individual spikes of various characteristics. The data were collected during $1990.4 \mathrm{~h}$ of observations in 357 observing days between February 2000 and December 2001. Using X-ray data taken with Yohkoh, GOES and Interball satellites, UV data taken with TRACE and $\mathrm{SOHO}$ satellites and ground based observations made in the $\mathrm{H} \alpha$ hydrogen line we have identified the most likely active solar phenomena related to each radio event with spikes as well as investigating the main morphological properties and evolution of the spikes.

Eleven groups of spikes were emitted during the increasing phase of the solar flares, two groups were emitted during the peak of the flares and five groups were observed during the 


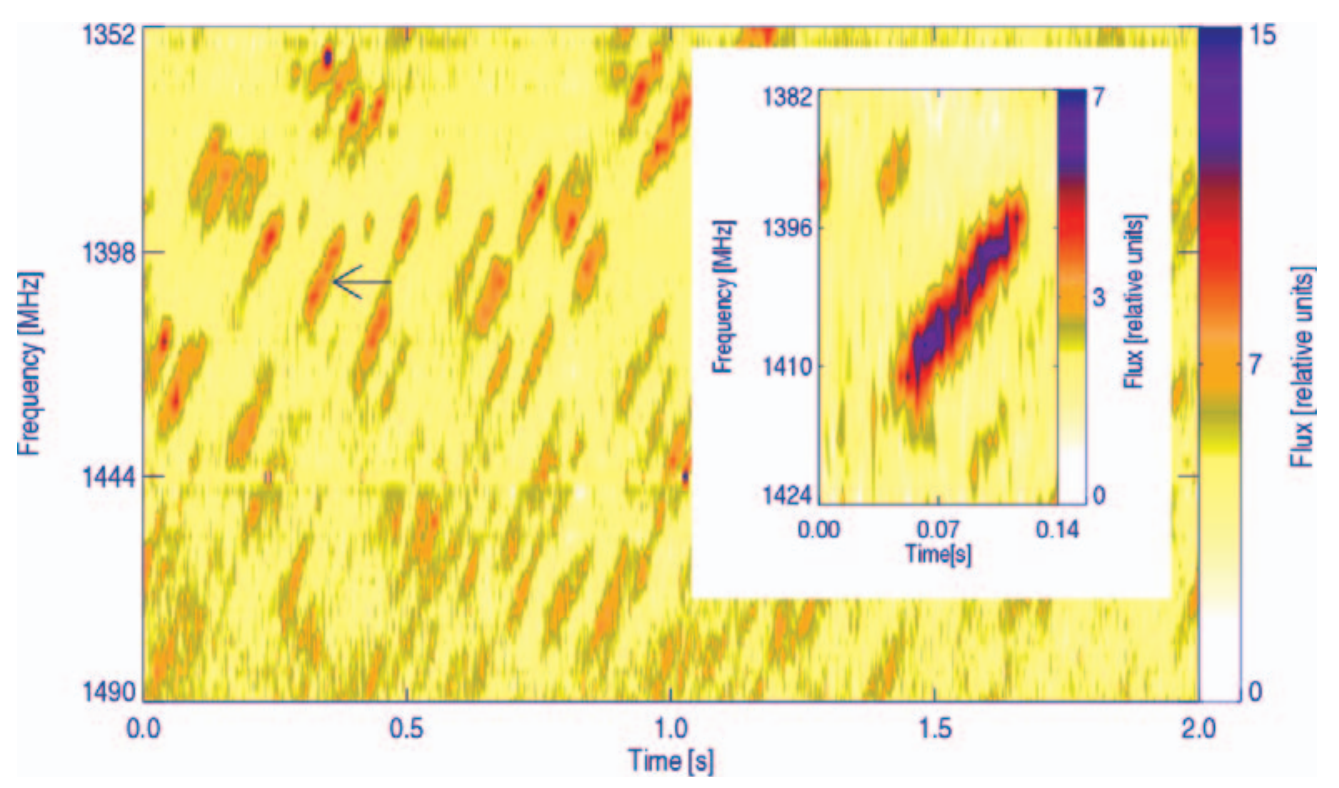

Fig. 19. Dynamic radiospectrogram of the dm radio spikes recorded on 30 October 2001, beginning at 12:20:27 UT.
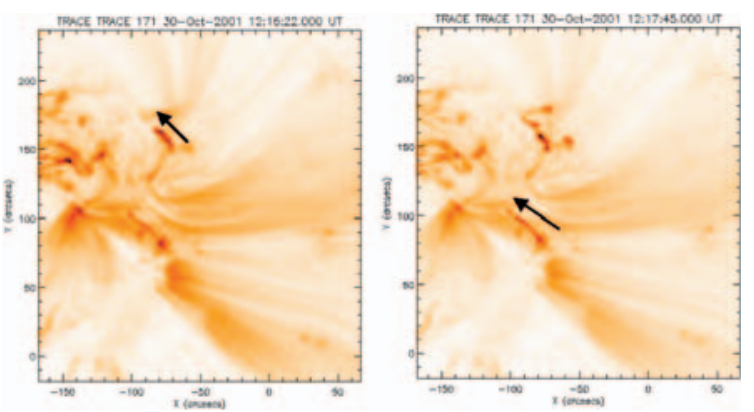

Fig. 20. Images of the NOAA 9682 active region taken with TRACE $(171 \AA)$ on 30 October 2001 at 12:16:22 UT (left panel) and 12:17:45 UT (right panel). The arrows show the direction of the plasma motion.

gradual phase of the flares (according to the GOES flux curves for the 1-8 $\AA$ band). Using the Yohkoh/HXT flux curves made in L and M1 channels, we found that six of eleven groups of spikes observed during the increasing phases undoubtedly occurred during the impulsive phase of the relevant flare. Five groups of $\mathrm{dm}$ radio spike emission were correlated in time with slow increases of the X-ray flux, leading to the solar flare. Using the GOES database, we found that no more than $2 \%$ of the solar flares were associated with radio spikes. However, probably all spikes are emitted as a result of some process connected with solar flares.

The analysis of the available observing materials shows that the emission of the $\mathrm{dm}$ radio spikes can be initiated in various configurations of the interacting magnetic fields and can be connected with various interactions between plasma and magnetic fields: interactions of the magnetic loops of Y and I configurations, bulk motions (ejections) of the plasma along long magnetic loops as well as rebuilding of the arcade of loops.

On the dynamic radiospectrograms of the investigated events we have found two kinds of well outlined structures formed by groups of individual spikes: chains of spikes and the different structures referred to as "columns". The columns of spikes are formed by numerous individual spikes, emitted in a very short time range on various frequency in a whole observed band. In a frame of the column each individual spike can show its own positive (i.e. from low to high) or negative (i.e. from high to low) drift in frequency. The clear differences of the temporal evolution between chains and columns of spikes can be attributed to the differences in the structure of the radio sources.

We have evaluated the main observational parameters of the 5328 individual $\mathrm{dm}$ radio spikes. The shortest observed spike lasted $0.0026 \mathrm{~s}$ only, the longest one $0.284 \mathrm{~s}$. The distribution of the spike durations is right-skewed with skewness equal to 1.64 , its median is equal to $0.029 \mathrm{~s}$, the mean is $0.036 \mathrm{~s}$. 5165 spikes $(97 \%)$ were shorter than $0.1 \mathrm{~s}$, fulfilling the commonly accepted upper limit on spike duration. The presented durations of the spikes are much longer than mean duration of the spikes, equal to $10 \mathrm{~ms}$, estimated for the same frequency band by Güdel \& Benz (1990), and also are longer than the $10 \mathrm{~ms}$ mean duration of the spikes observed by Mészárosová et al. (2003). The differences cannot be attributed to the limited temporal resolution of the collected data.

Thanks to the very high temporal resolution of the radiospectrograph used, we have found numerous individual $\mathrm{dm}$ radio emissions with visible subtle structure of the timefrequency distribution of the emission intensity; their dynamic spectrograms are strongly heterogeneous and fragmented. We have found that in a frame of the single radio event all emitted $\mathrm{dm}$ radio spikes are rather similar. Nevertheless, both types of spikes (with or without internal structure) can be emitted in the same event. Each spike has its own positive (i.e. from low to high) or negative (i.e. from high to low) drift in frequency of various speed (from tens of $\mathrm{MHz}$ per second up to greater than the highest measurable with our instrument, equal to $37.5 \mathrm{GHz}$ per second). If these emissions are genuine, individual spikes with real internal subtle structure or if they are in fact very short 


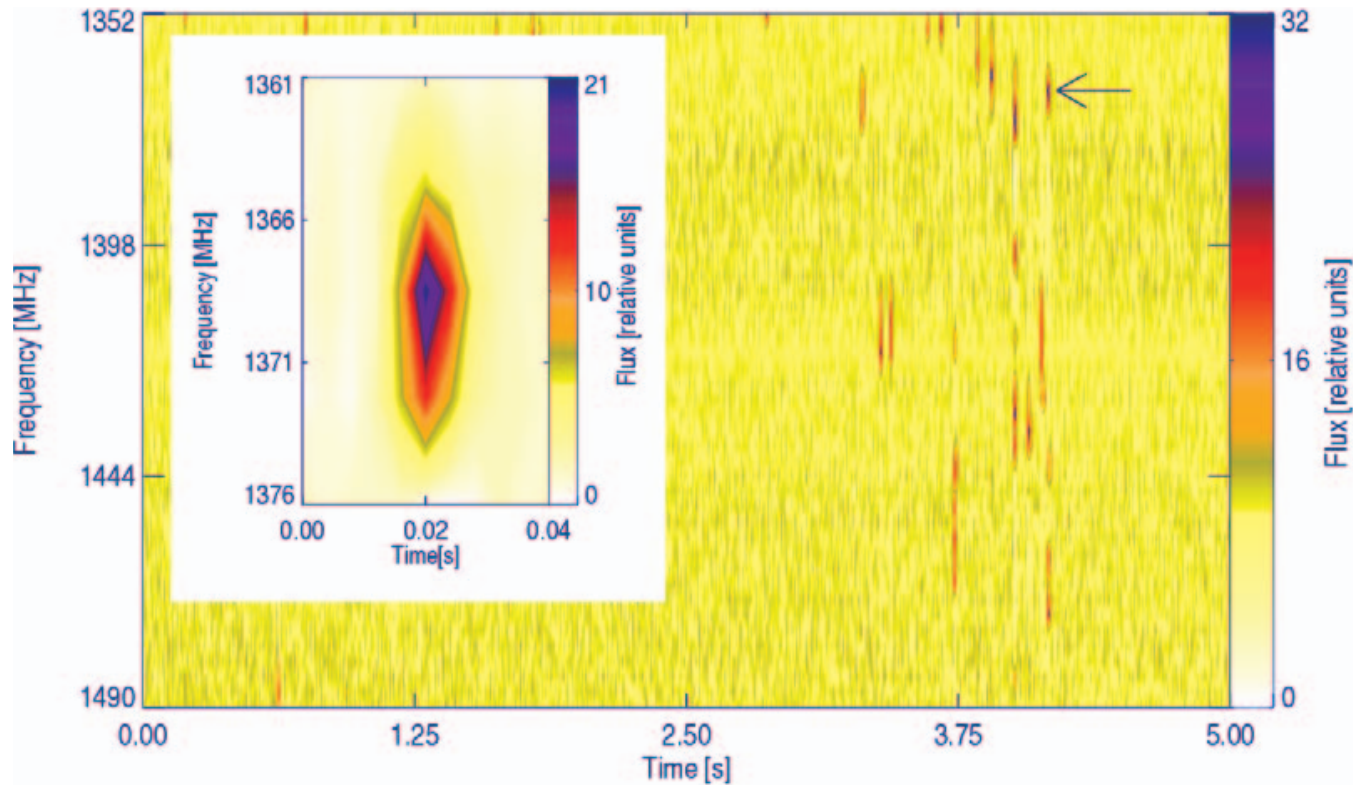

Fig. 22. Dynamic radiospectrogram of the second group of dm radio spikes recorded on 11 July 2000, beginning at 13:27:25 UT.

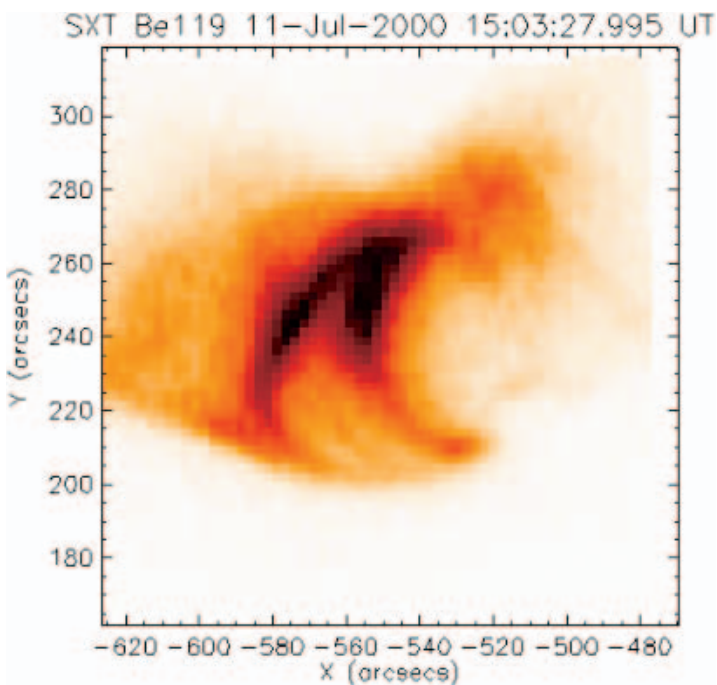

Fig. 25. Image of the NOAA 9077 active region taken with SXT/Be119 telescope on 11 July 2000 at 15:03:28 UT.

lasting chains of tightly packed spikes with fast drifts in frequency should be addressed by future investigations.

The distribution of the durations of the 1699 spikes without internal structure shorter than $0.1 \mathrm{~s}$ is right-skewed with skewness equal to 1.86 , its median is equal to $0.024 \mathrm{~s}$ and its mean is equal to $0.029 \mathrm{~s}$. The distribution of durations of the 1837 spikes with internal structure shorter than $0.1 \mathrm{~s}$ is close to Gaussian (skewness equal to 0.12 ) with the median equal to $0.047 \mathrm{~s}$ and the mean equal to $0.046 \mathrm{~s}$. The relative widths in frequency of these emissions are similar to the parameters of the "classical" spikes without internal structure.

Taking into account all 13 events, seven events have a majority of spikes with negative drifts, five events have almost the same numbers of spikes with positive and negative drifts.

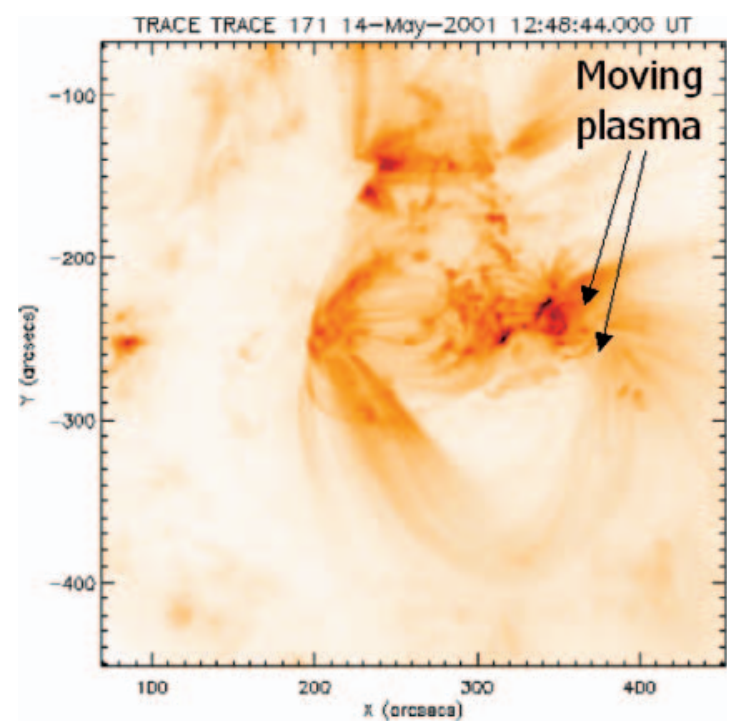

Fig. 27. Images of the NOAA 9455 active region taken with TRACE (171 ̊) on 14 May 2001 at 12:48:44 UT.

2769 spikes (52\%) have negative drifts, 760 (14\%) have positive drifts, the remaining spikes (1799) have immeasurable drifts. The drifts of the spikes with internal structure are distinctly slower than ones without structure.

The more extended results of the statistical investigation of the whole population of spikes as well as the properties of the individual spikes will be published in a separate paper (in preparation).

Acknowledgements. We are grateful to Dr G. D. Fleishman for many helpful remarks and comments during the writing of the paper. PR and MS were supported by the Polish State Committee for Scientific Research (KBN) grant number PBZ KBN 054/P03/2001, RF was supported by the KBN grant number 2 PO3D 00123. 


\section{References}

Allaart, M. A. F., van Nieuwkoop, J., Slottje, C., \& Sondaar, L. H. 1990, Sol. Phys., 130, 183

Aschwanden, M. J. 1990, A\&A, 237, 512

Aschwanden, M. J., \& Güdel, M. 1992, ApJ, 401, 736

Aschwanden, M. J., \& Benz, A. O. 1997, ApJ, 480, 825

Bárta, M., \& Karlický, M. 2001, A\&A, 379, 1045

Bastian, T. S., Benz, A. O., \& Gary, D. E. 1998, ARA\&A, 36, 131

Benz, A. O. 1985, Sol. Phys., 96, 357

Benz, A. O. 1986, Sol. Phys., 104, 99

Benz, A. O. 2002, Solar and Stellar Coronae (Kluwer Academic Publishers)

Benz, A. O., \& Kane, S. R. 1986, Sol. Phys., 104, 179

Benz, A. O., Messmer, P., \& Monstein, C. 2001, A\&A, 366, 326

Benz, A. O., Saint-Hilaire, P., \& Vilmer, N. 2002, A\&A, 383, 678

Cadwell, B. J. 1997, Pulsar Survey And Timing With The Pann State Pulsar Machines, Ph.D. Thesis

Csillaghy, A., \& Benz, A. O. 1993, A\&A, 274, 487

Dạbrowski, B. P., Zajączkowski, R., \& Kus, A. J. 2002, ESA SP-505, 381

Dạbrowski, B. P., Rompolt, B., Falewicz, R., et al. 2003, ESA SP-535, 491

De Groot, T. 1962, Inf. Bull. Solar Radio Obs. Europe, 9, 3

Delaboudinière, J.-P., Artzner, G. E., Brunaud, J., et al. 1995, Sol. Phys., 162, 291

Dröge, F. 1967, Astrophys., 66, 176

Dröge, F. 1977, A\&A, 57, 285

Dröge, F., \& Riemann, P. 1961, Inf. Bull. Solar Radio Obs. Europe, 8, 6

Elgarøy, Ø. 1961, ApNr., 7, 123

Fleishman, G. D., \& Yastrebov, S. G. 1994, Sol. Phys., 154, 361

Fleishman, G. D., \& Melnikov, V. F. 1999, ESA SP-448, 1247
Fleishman, G. D., \& Arzner, K. 2000, A\&A, 358, 776

Fleishman, G. D., Gary, D. E., \& Nita, G. M. 2003, ApJ, 593, 571

Gary, D. E., Hurford, G. J., \& Flees, D. J. 1991, ApJ, 369, 255

Güdel, M., \& Benz, A. O. 1988, A\&AS, 75, 243

Güdel, M., \& Benz, A. O. 1990, A\&A, 231, 202

Güdel, M., \& Zlobec, P. 1991, A\&A, 245, 299

Holman, G. D., Eichler, D., \& Kundu, M. R. 1980, Radio physics of the Sun; Proceedings of the Symposium, College Park, Md., August 7-10, 1979. A80-53501 24-92 (Dordrecht: D. Reidel Publishing Co.), 457

Jin, S.-Z., Zhao, R.-Y., \& Fu, Q.-J. 1986, Sol. Phys., 104, 391

Karlický, M., Sobotka, M., \& Jiřička, K. 1996, Sol. Phys., 168, 375

Karlický, M., Yan, Y., Fu, Q., et al. 2001, A\&A, 369, 1104

Kosugi, T., Makishima, K., et al. 1991, Sol. Phys., 136, 17

Kuijpers, J., van der Post, P., \& Slottje, C. 1981, A\&A, 103, 331

Kuznetsov, A. A., \& Vlasov, V. G. 2003, Astron. Rep., 47, 129

Melrose, D. B., \& Dulk, G. A. 1982, ApJ, 259, 844

Messmer, P., \& Benz, A. O. 2000, A\&A, 354, 287

Mészárosová, H., Veronig, A., Zlobec, P., et al. 2003, A\&A, 407, 1115

Robinson, P. A. 1991, Sol. Phys., 134, 299

Rudawy, P., Rompolt, B., Falewicz, R., Da̧browski, B. P., \& Siarkowski, M. 2002, ESA SP-506, 741

Sakai, J., \& de Jager, C. 1996, Space Sci. Rev., 77, 1

Stähli, M., \& Magun, A. 1986, Sol. Phys., 104, 117

Sylwester, J., Fárník, F., Likin, O., et al. 2000, Sol. Phys., 197, 337

Tajima, T., Benz, A. O., Thaker, M., \& Leboeuf, J. N. 1990, ApJ, 353, 566

Tsuneta, S., Acton, L., Bruner, M., et al. 1991, Sol. Phys., 136, 37

Sharma, R. R., \& Vlahos, L. 1984, ApJ, 280, 405

Wang, S. J., Yan, Y. H., \& Fu, Q. J. 2002, Sol. Phys., 209, 185

Wentzel, D. G. 1991, ApJ, 373, 285

Winglee, R. M., Dulk, G. A., \& Pritchett, P. L. 1988, ApJ, 328, 809

Wolfson, J., Bruner, M., Jurcevich, B., et al. 1997, BAAS, 29, 887 
B. P. Dạbrowski et al.: Millisecond radio spikes in the decimetre band and their related active solar phenomena, Online Material p 1

\section{Online Material}


B. P. Da̧browski et al.: Millisecond radio spikes in the decimetre band and their related active solar phenomena, Online Material p 2

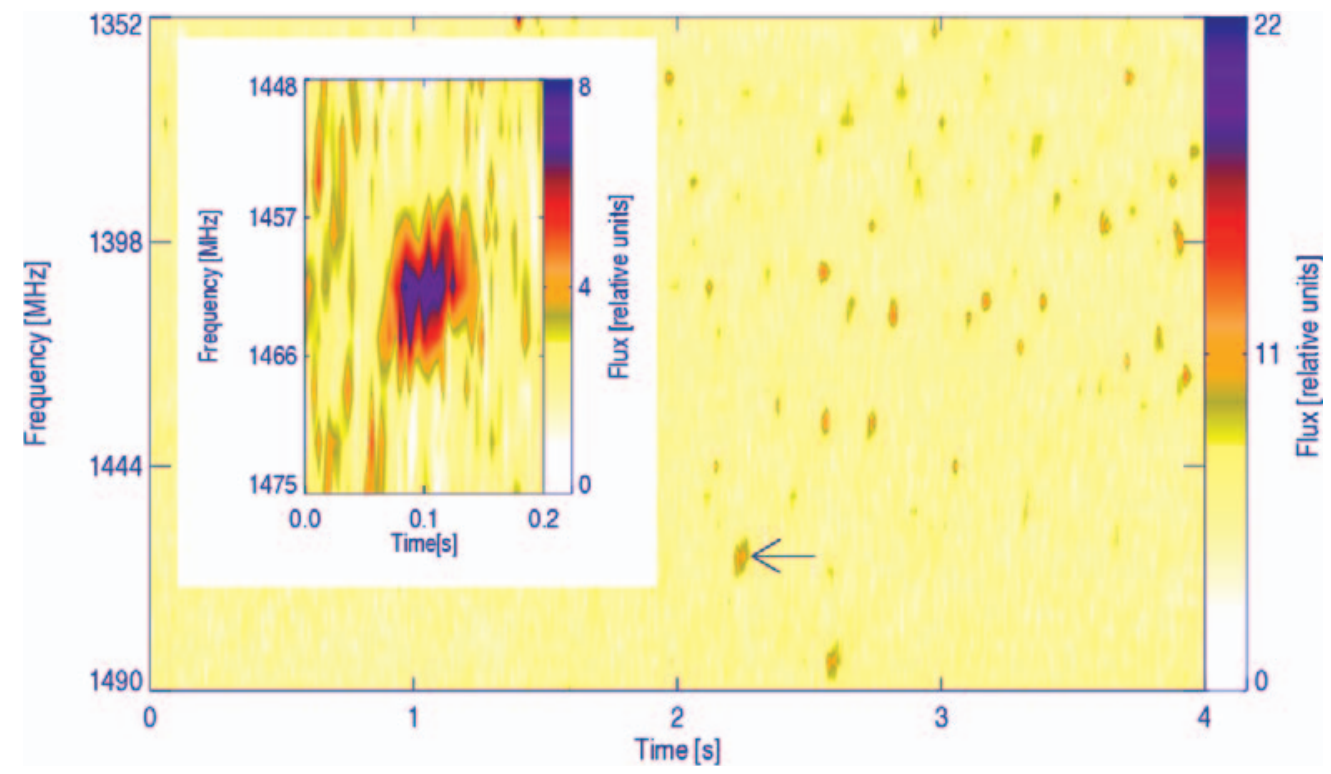

Fig. 3. Dynamic radiospectrogram of the dm radio spikes recorded on 15 March 2000, beginning at 12:20:40 UT. Some spikes form clearly visible chains. Here and in the next radiospectrograms the flux is scaled in relative units, the arrow point the spike presented in detail.

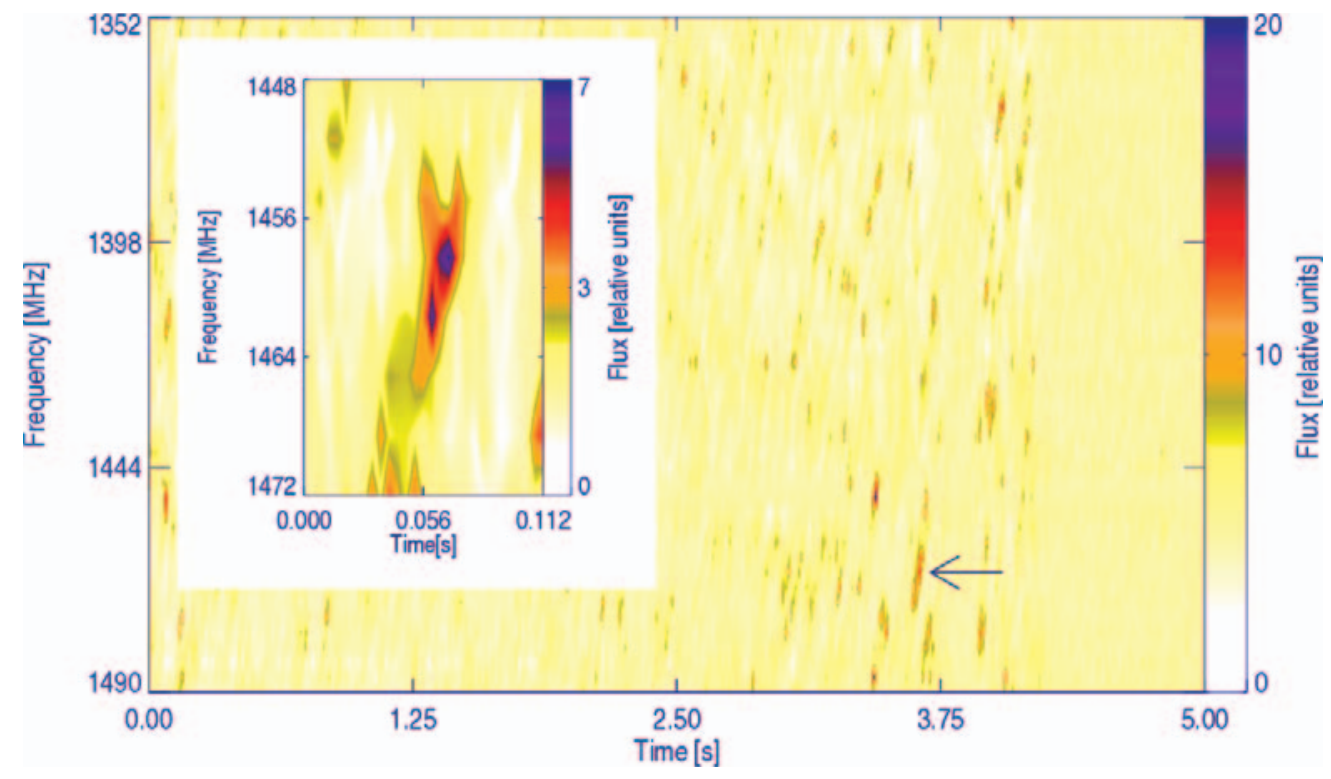

Fig. 5. Dynamic radiospectrogram of the dm radio spikes recorded on 21 June 2000, beginning at 09:25:45 UT. One can notice both types of spikes: with and without internal structure; some of them are aggregated in columns of spikes. 
B. P. Dąbrowski et al.: Millisecond radio spikes in the decimetre band and their related active solar phenomena, Online Material $p 3$

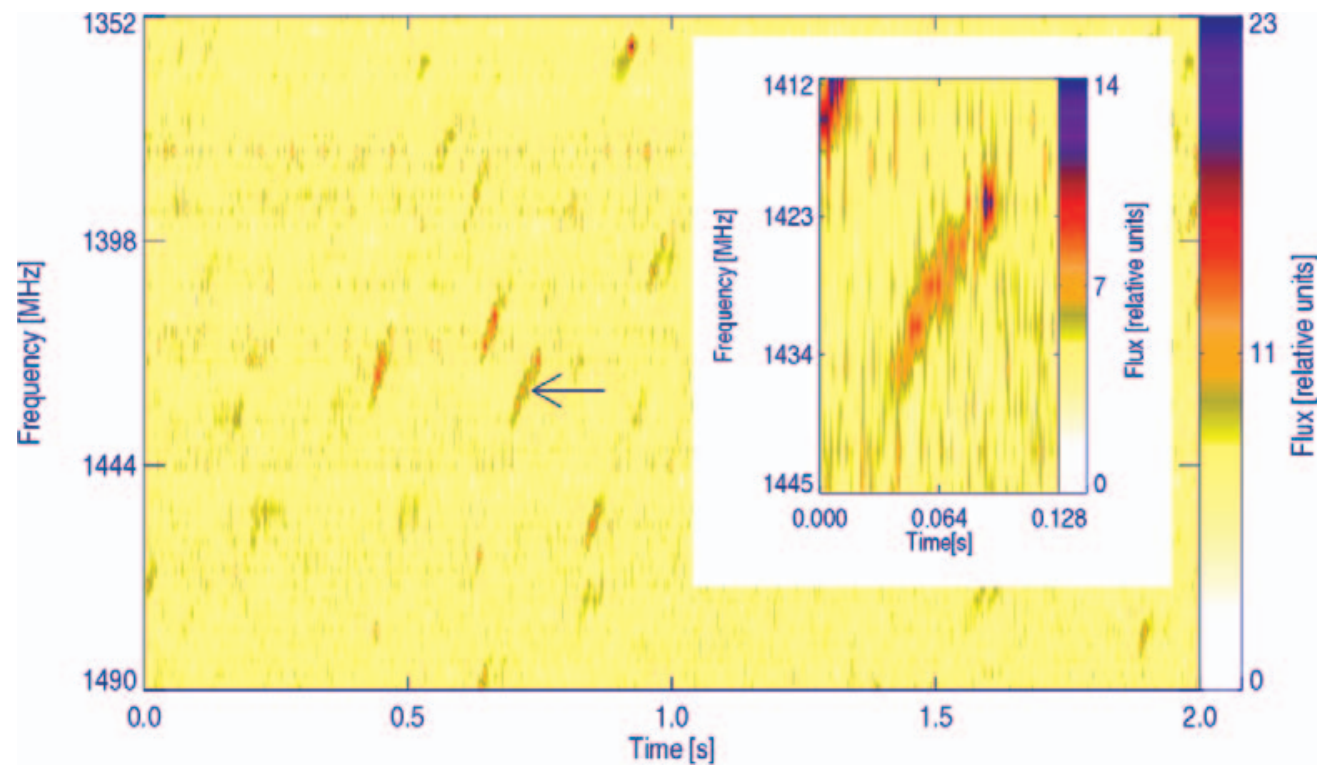

Fig. 9. Dynamic radiospectrogram of the dm radio spikes recorded on 23 April 2001, beginning at 10:14:58 UT.

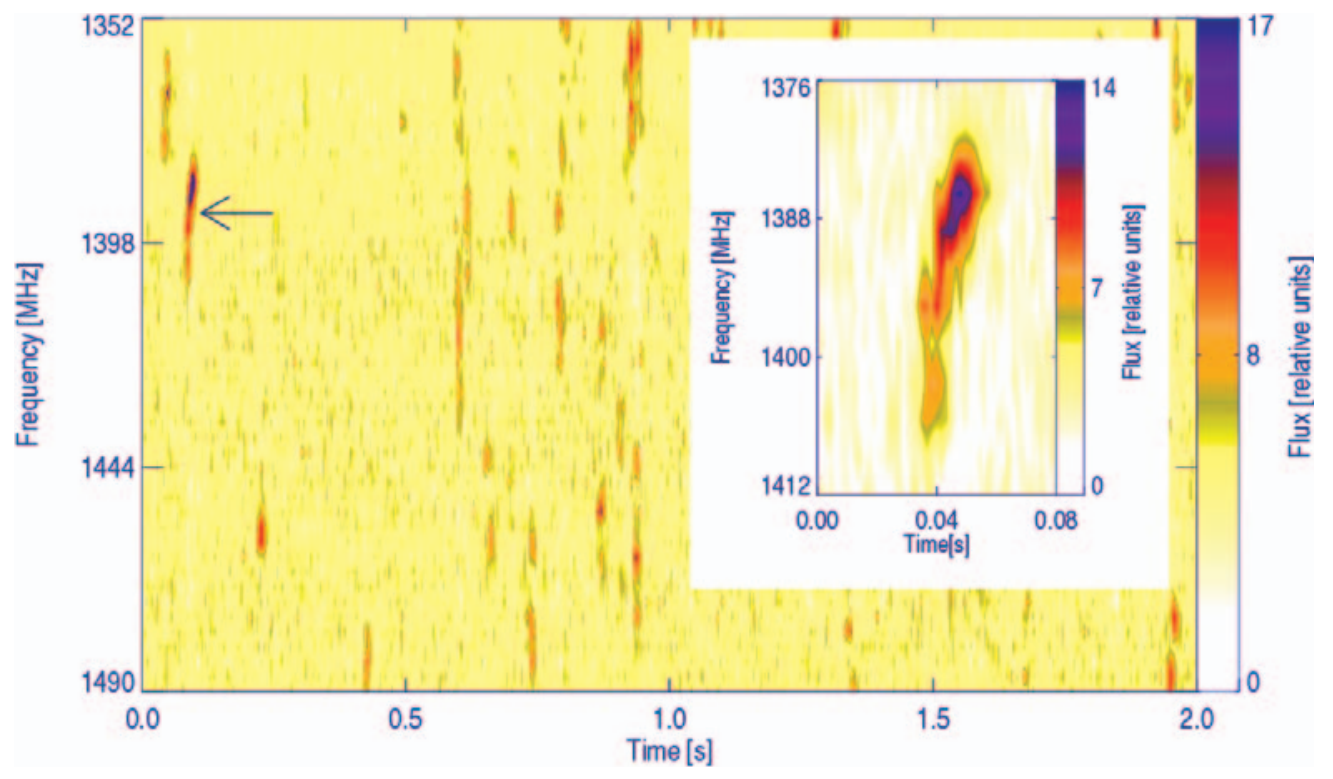

Fig. 15. Dynamic radiospectrogram of the dm radio spikes recorded on 21 May 2000, beginning at 09:39:28 UT. 
B. P. Dạbrowski et al.: Millisecond radio spikes in the decimetre band and their related active solar phenomena, Online Material p 4

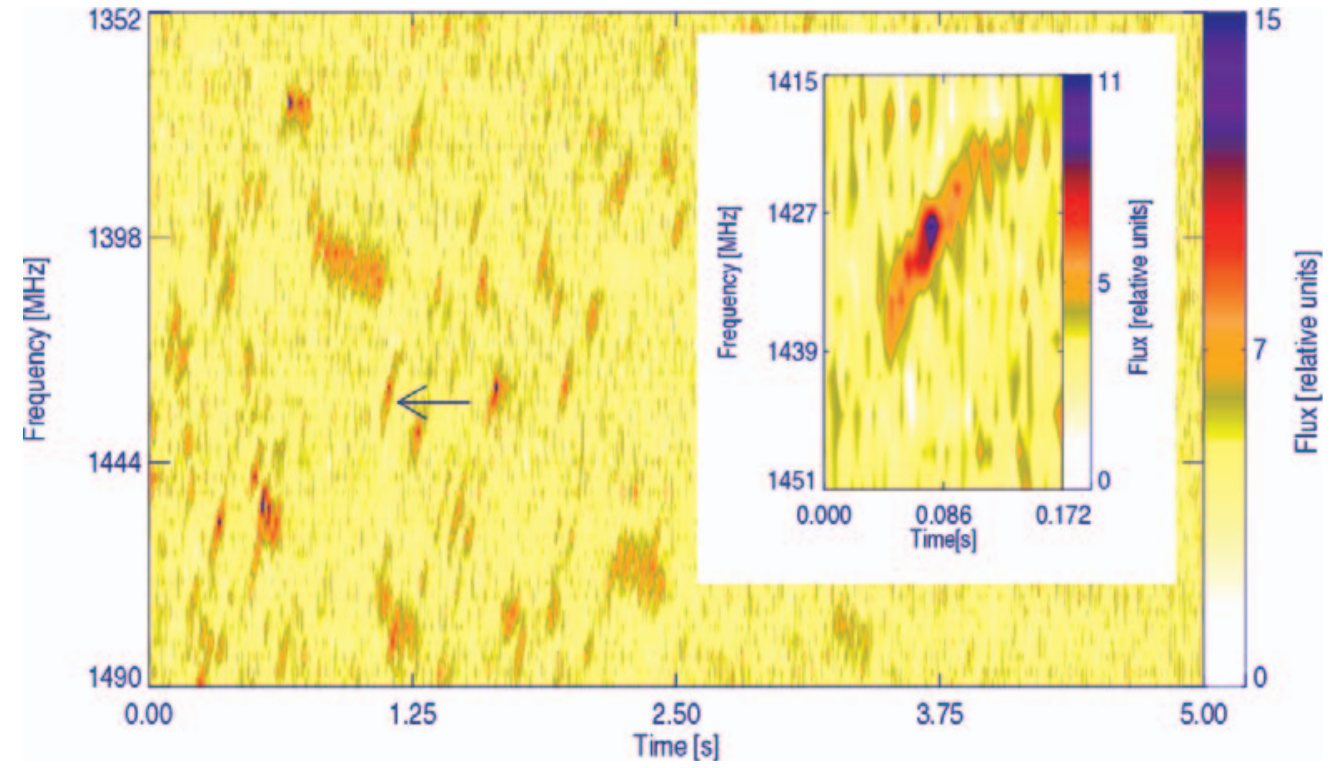

Fig. 16. Dynamic radiospectrogram of the dm radio spikes recorded on 26 March 2000, beginning at 10:38:00 UT. Some spikes show clearly visible internal subtle structure.

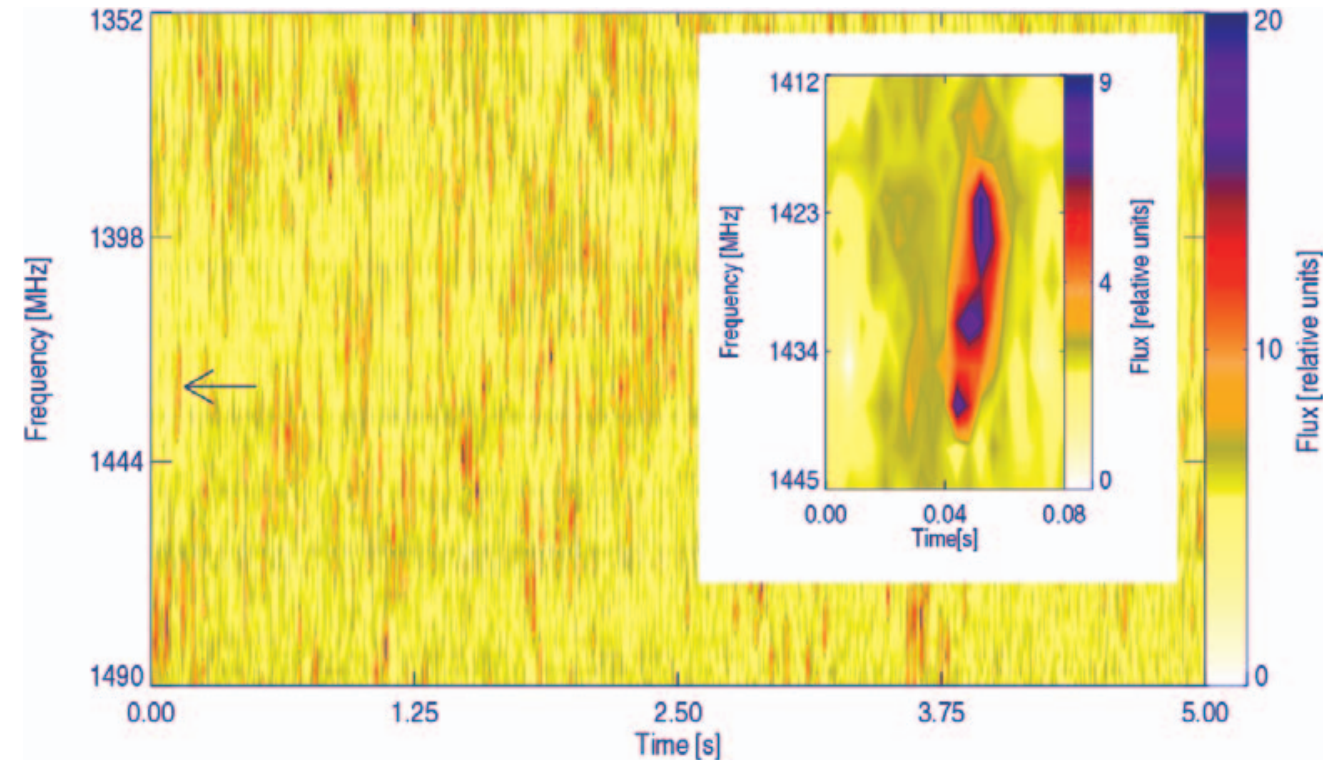

Fig. 21. Dynamic radiospectrogram of the first group of dm radio spikes recorded on 11 July 2000, beginning at 13:16:20 UT. 
B. P. Dąbrowski et al.: Millisecond radio spikes in the decimetre band and their related active solar phenomena, Online Material p 5

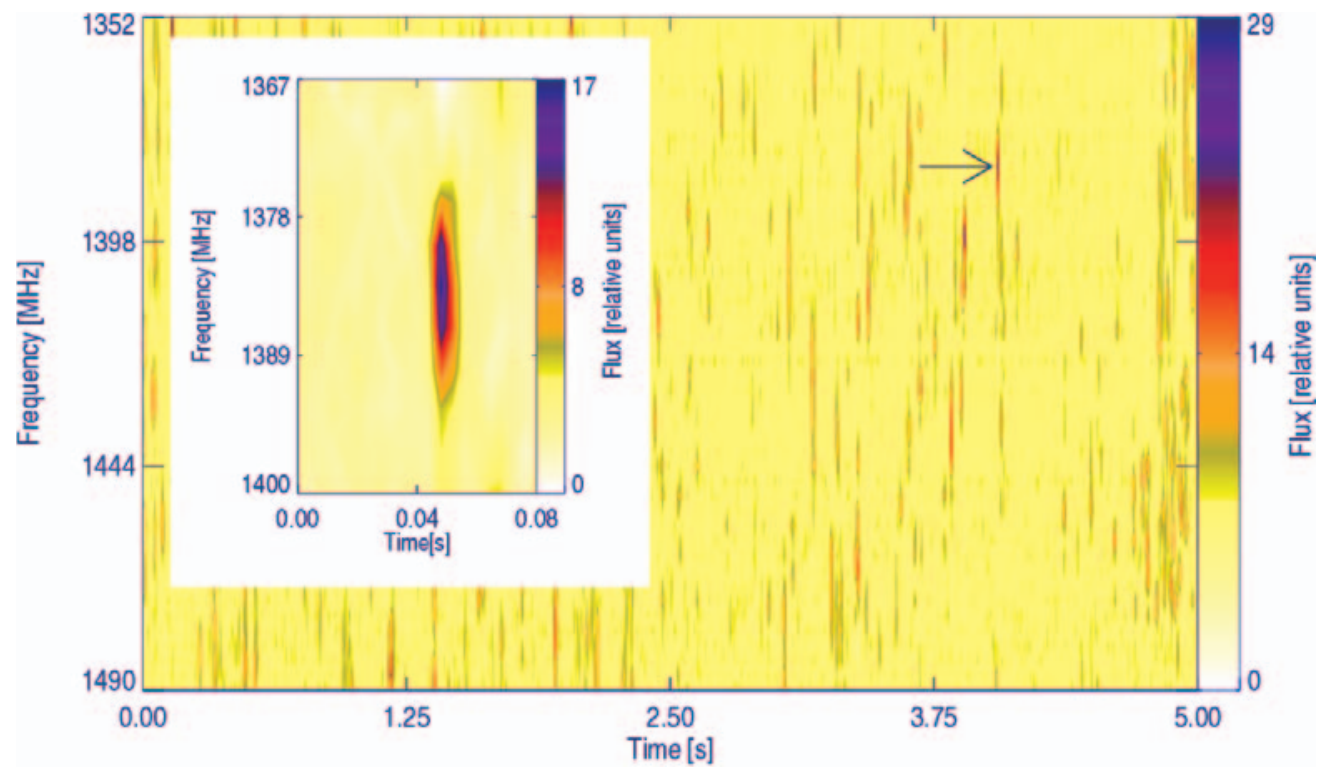

Fig. 23. Dynamic radiospectrogram of the third group of dm radio spikes recorded on 11 July 2000, beginning at 14:43:10 UT.

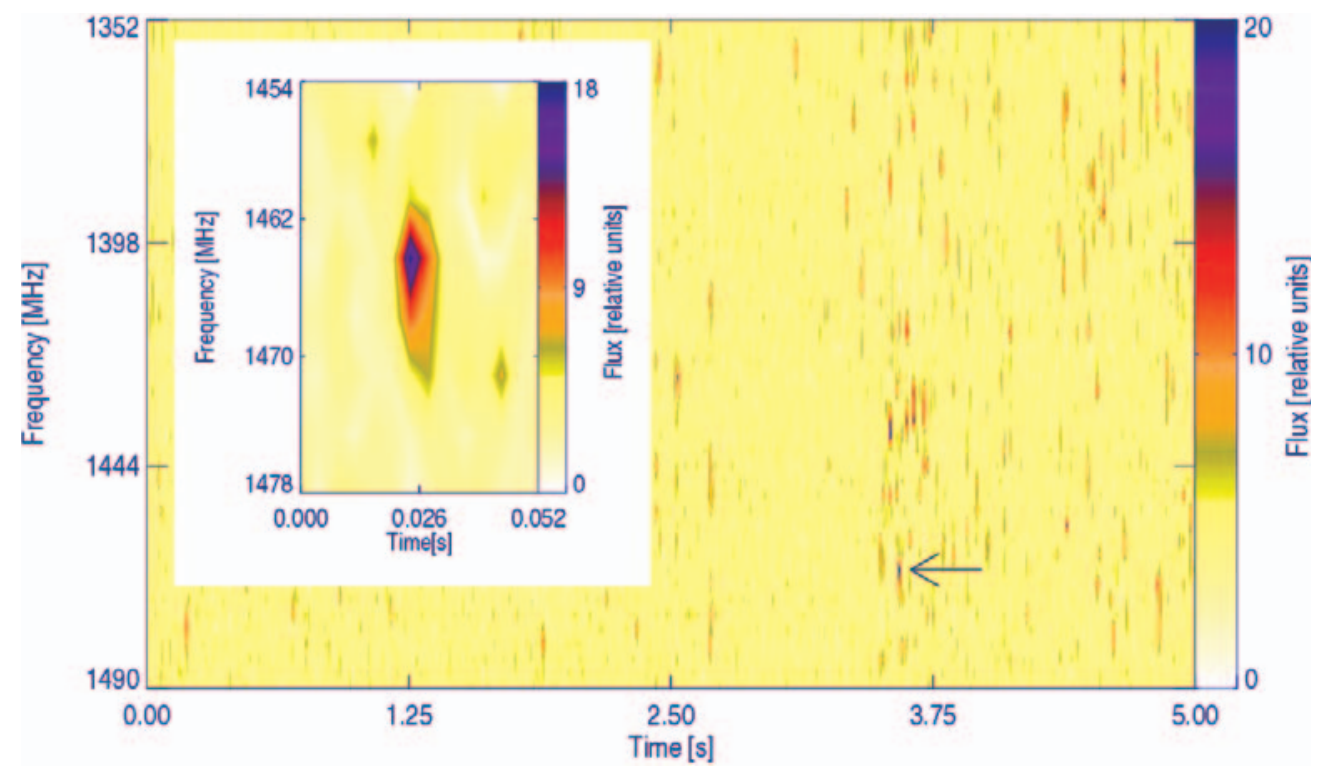

Fig. 24. Dynamic radiospectrogram of the fourth group of dm radio spikes recorded on 11 July 2000, beginning at 14:59:05 UT. 
B. P. Dạbrowski et al.: Millisecond radio spikes in the decimetre band and their related active solar phenomena, Online Material p 6

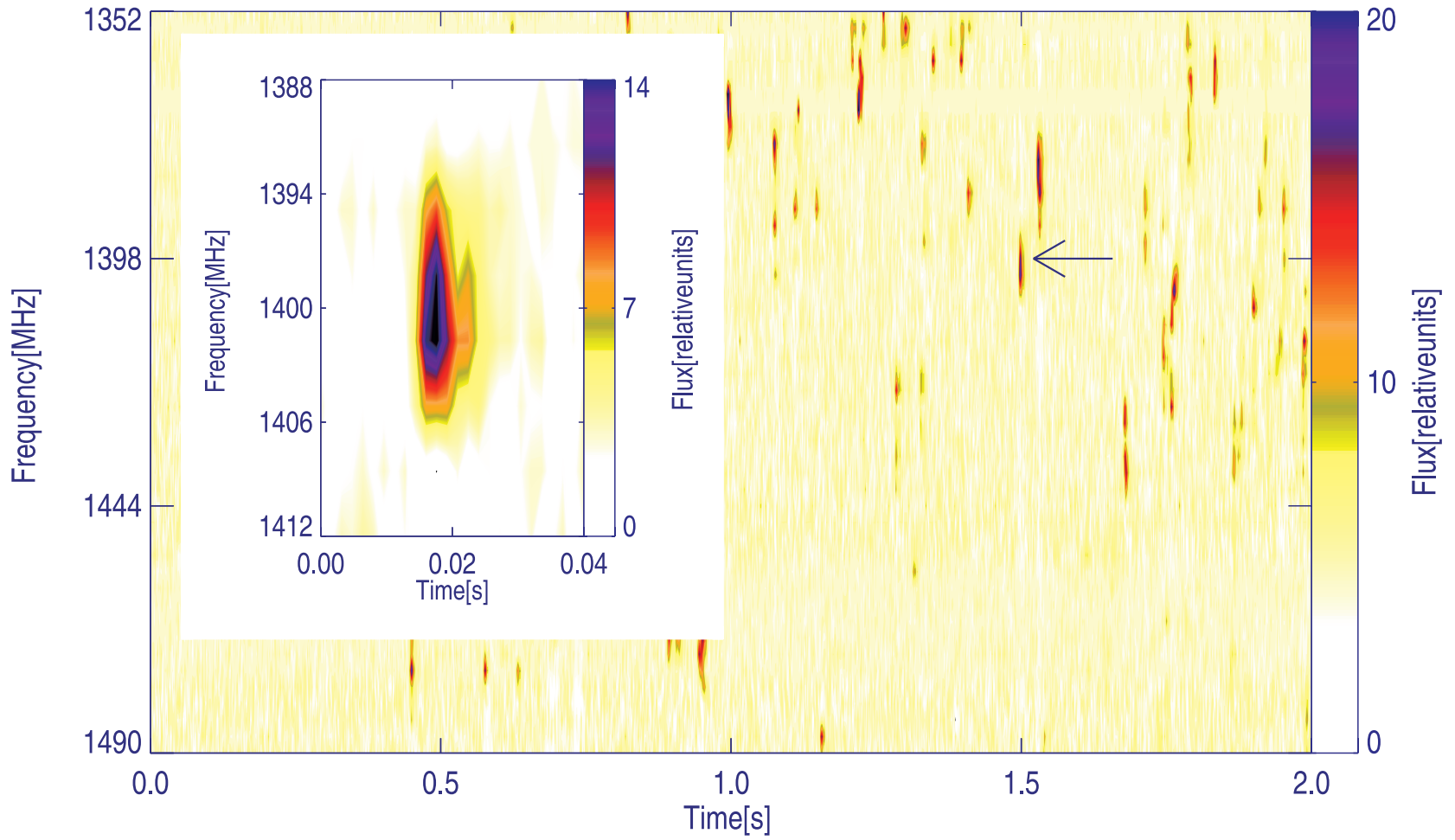

Fig. 26. Dynamic radiospectrogram of dm radio spikes recorded on 14 May 2001, beginning at 12:47:29 UT. 